JOURNAL OF ETHNOBIOLOGY

AND ETHNOMEDICINE

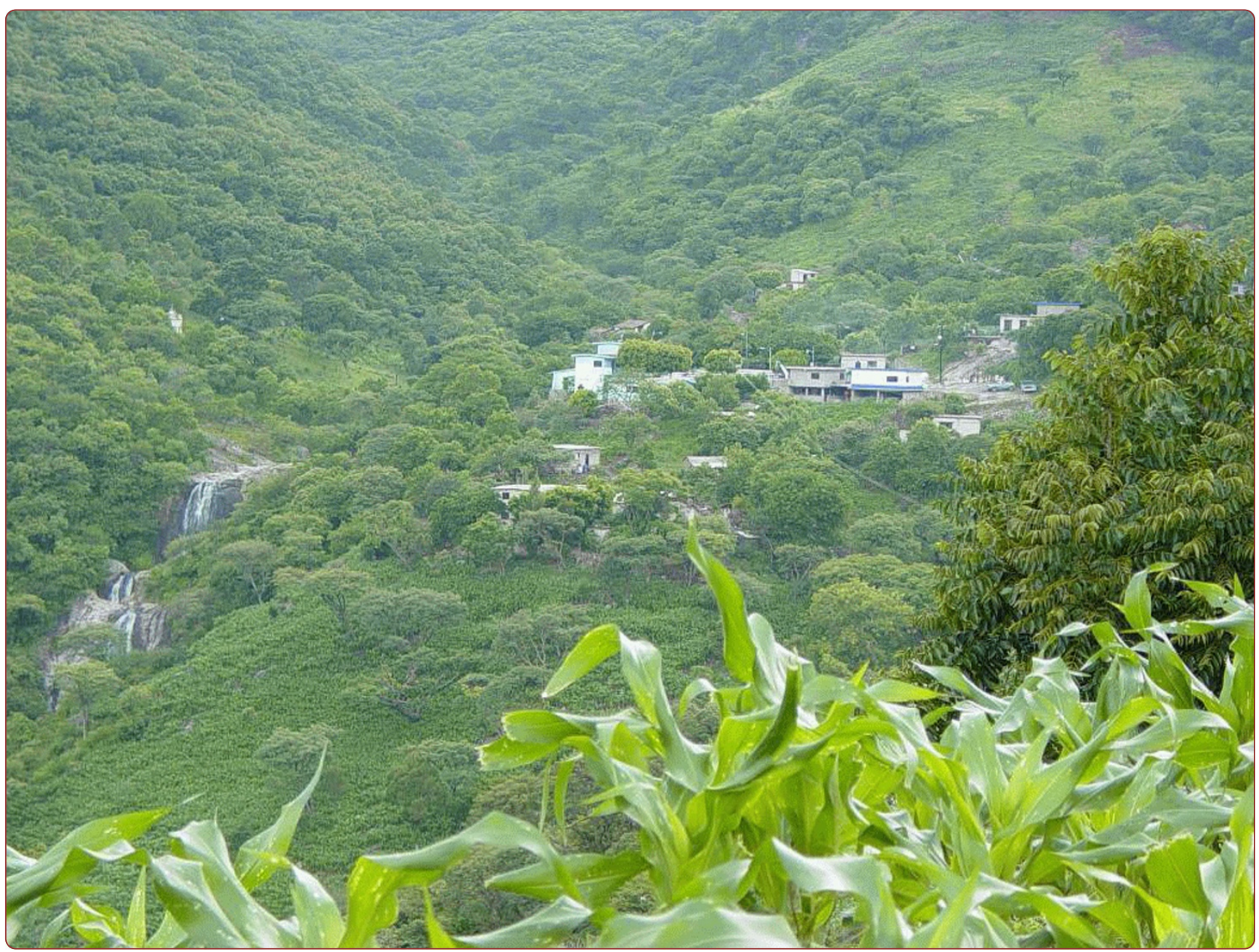

\title{
Factors affecting ethnobotanical knowledge in a mestizo community of the Sierra de Huautla Biosphere Reserve, Mexico
}

Beltrán-Rodríguez et al. 


\title{
Factors affecting ethnobotanical knowledge in a mestizo community of the Sierra de Huautla Biosphere Reserve, Mexico
}

Leonardo Beltrán-Rodríguez ${ }^{1,2 *}$, Amanda Ortiz-Sánchez ${ }^{3}$, Nestor A Mariano ${ }^{3}$, Belinda Maldonado-Almanza ${ }^{3}$ and Victoria Reyes-García ${ }^{4}$

\begin{abstract}
Background: Worldwide, mestizo communities's ethnobotanical knowledge has been poorly studied. Based on a mestizo group in Mexico, this study assesses a) the use value (UV) of the local flora, b) gendered differences in plant species, and c) the association between socio-economic variables and ethnobotanical knowledge.

Methods: To assess the degree of knowledge of plant resources, we conducted 41 interviews collecting information on knowledge of local plant resources and the socio-economic situation of the informant. We also collected free listings of useful plants by category of use to identify the UV of each species. With the support of key informants, we photographed and collected the plant material recorded during the interviews and free listings on five different habitats. Paired t-tests and a Wilcoxon signed rank test were used to determine differences in the number of species known by men and women. Differences in distribution were analyzed by means of the Shapiro-Wilk's W normality tests. To determine the association of socio-economic factors and ethnobotanical knowledge, we used a non-metric multidimensional scaling analysis (NMDS).

Results: Informants listed 185 species. Medicinal plants constituted the most diverse group (90 species). Tropical deciduous forest is the habitat that concentrates the highest proportion of plant resources (80 species). The use-values were classified into three groups: A (4-6 UV; three species), B (0.35-1.37 UV; 39 species) and C (0-0.29 UV; 143 species). High-quality wood species and those associated to religious ceremonies had the highest UV. Women's and men's knowledge of plant species showed statistically significant differences at the interspecific and the intracategorical levels (Student's test, T15 $=4.8, \mathrm{p}<0.001$ ). Occupation, gender and age were statistically significant associated to ethnobotanical knowledge $(p<0.05)$, whereas income, education level, and place of origin were not.

Conclusion: This research improves our understanding of the socio-economic activities associated with the intracultural distribution of ethnobotanical knowledge among mestizo Mexican communities. It also provides information on plant resources and habitats and how local peasants value them. This information could help in the development of proposals to improve biocultural conservation and strengthen traditional knowledge systems for effective forest management.
\end{abstract}

Keywords: Ethnobotanical knowledge, Socio-economic variables, Mestizo community, Mexico, Useful plants

\footnotetext{
*Correspondence: beltran.leonardo@colpos.mx

${ }^{1}$ Facultad de Ciencias Biológicas, Universidad Autónoma del Estado de

Morelos, Av. Universidad № 1001, Cuernavaca CP: 62210, Mexico

${ }^{2}$ Present address: Postgrado en Botánica, Colegio de Postgraduados,

Montecillo, Texcoco C.P. 56230, México

Full list of author information is available at the end of the article
} 


\section{Background}

Traditional knowledge, understood as cumulative body of knowledge, practices and beliefs about the environment evolving by adaptive processes and handed down through generations by cultural transmission $[1,2]$, has been widely documented in diverse Mesoamerican groups. Most of this research has focused on indigenous communities located in Mexico. In Mexico, studies have approached different fields of traditional knowledge including the domestication of plants $[3,4]$, the folk classification of the natural world [5], the cultural meaning of wild species [6], the loss and changes of knowledge [7,8], the use and management of wild species, e.g., food, timber, textiles, fuels and others $[9,10]$, particularly the knowledge of medicinal plants [11].

It has been argued that the use of plants in indigenous communities is associated to biological, ecological and socio-cultural factors, including production techniques and practices, religion, gender, and age [12-16]. Such aspects have been extensively studied in several indigenous societies in other parts of the world [12-14,17], highlighting the different patterns in knowledge distribution and loss, leading to different changes in the use and management of such resources. However, researchers have paid scant attention to the association between such socio-cultural and socio-economics factors and the acquisition of traditional knowledge in mestizo communities see $[15,18,19]$, for some exceptions.

The study of the traditional knowledge of mestizo communities is important because such communities account for about $75 \%$ of Mexico forested surface [20], so understanding the process of knowledge acquisition in those communities, as well as what are the species with larger use value can have important implications for the conservation and management of forested resources [21]. An additional reason to focus on mestizo communities is that their knowledge seems to be different from that of indigenous societies. Although some studies in Mexico do not show significant differences between indigenous and mestizo communities [22], other studies suggest that indigenous communities use more frequently medicinal, edible, and firewood plant species, whereas mestizo communities use more frequently plant species for construction [23].

Previous ethnobotanical studies carried out with mestizo groups in Mexico have been mainly oriented to making inventories of useful plants at specific locations [24], although the focus has recently changed and nowadays researchers are more interested in examining how ecological and social aspects (i.e., occupation, education level, gender, degree of urbanization, or relation with other communities) shape traditional knowledge [22,25-27].

In this context, we conducted a research in the mestizo community of El Salto, Morelos, Mexico. The research aimed to answer the following questions: 1) Which plant species do mestizo people use to satisfy their needs? 2) Which is the use-value of the local flora? 3) Are socio-economic variables associated to the acquisition of ethnobotanical knowledge in a mestizo community?.

Researchers have previously studied the association between ethnobotanical knowledge and socio-economic factors. Among the factors previously studied researchers have focused on the age [12,27-30], sex [6,12] 115 [29-31], the educational level [15,25,30], origin $[32,33]$, and the occupation and the wealth $[34,35]$ of informants. Among those, researchers have found that those having a stronger influence on shaping ethnobotanical knowledge distribution are age, sex, education level and wealth.

For example, several studies have found a positive association between age and traditional ethnobotanical knowledge [12], although some other studies have not found such association [15]. In contrast, the differences in ethnobotanical knowledge between men and women seem to be more consistent, with studies finding that men have a larger knowledge than women [13,15,29,31], although the trend seems to be inverse in relation to medicinal plants [30]. Such differences are generally explained by sexual distribution of work $[6,36]$. Some research also suggest that ethnobotanical knowledge decreases with the increase of education $[7,25,27,37]$ and wealth $[34,35]$. Several of those characteristics are also linked to the process of acculturation and the loss of indigenous languages (among indigenous communities) $[7,27,37]$. Some studies highlight the importance of occupation on traditional knowledge [25,38]. Martínez-Ballesté et al. [25] find that larger involvement in agricultural activities resulted in a loss of traditional ecological knowledge, as a consequence of the environmental transformation and loss of biodiversity. In contrast, those activities more dependent on the natural environment are associated to maintenance of traditional knowledge.

Given those previous findings, we hypothesize that the distribution of traditional knowledge will be patterned across socio-economic characteristics. Specifically, we expect to find that men, older people, people born in the area, and poorer people will have higher levels of traditional knowledge than people without those characteristics. We also hypothesize that people whose occupation depends on the environment, like people who practice extensive agriculture and stockbreeding, might also have larger levels of traditional knowledge.

Understanding the dynamics of ethnobotanical traditional knowledge among mestizo groups will provide information that is relevant to ethnobotany in two ways. First, it will help to understand the relations between social processes and the use and management of plant resources. And second, it will enable the development of 
approaches to conserve wild species taking into account the patterns of plant knowledge based on the use value of plant resources. At the applied level, the traditional knowledge of mestizo communities constitutes one of the multiple manifestations of Mexico's cultural diversity, and it is considered to be of great importance in terms of biological conservation both nationally and internationally $[39,40]$. It is also relevant for the development of ecologically and economically feasible proposals of socially just rural development, aimed to the promotion of bio-cultural conservation $[2,41]$.

\section{Methods}

\section{Study area}

This study was carried out at El Salto, a rural community located in the southern area of the state of Morelos, Mexico, within the Biosphere Reserve Sierra de Huautla. Its territory belongs to the mountain range known as Sierra Madre del Sur and it is part of the northern extreme of the Sierra de Huitzuco in the state of Guerrero, known as Cerro Frío. It is located at an altitude of
$1,785 \mathrm{~m}$ between the parallels $18^{\circ} 20^{\prime} 30^{\prime} \mathrm{N}$ and $99^{\circ} 17^{\prime}$ $21^{\prime} \mathrm{W}$, and it encompasses approximately 500 ha [42] (Figure 1).

The settlement is located in a transitional area between tropical deciduous forest and an oak forest [43]. Community surrounding areas include modified environments such as home gardens and farming lands. The dominant climate is semi-warm, semi-humid, with rains during the summer. The total annual rainfall is $924.3 \mathrm{~mm}$ and the average annual temperature is $28^{\circ} \mathrm{C}$ [44].

Inhabitants of El Salto are mestizo. The community was founded by farmers from the southern state of Morelos and by some migrant communities and adjoining villages of the state of Guerrero during the Mexican Revolution (1910-1920). The community is made up of 108 residents that belong to 25 households and each household houses around five people. All families share kinship networks with others, so there is a core of coexistence and knowledge originated from this cause. There are 55 women and 53 men, but children and young people (between 1 and 25 years of age) outnumber

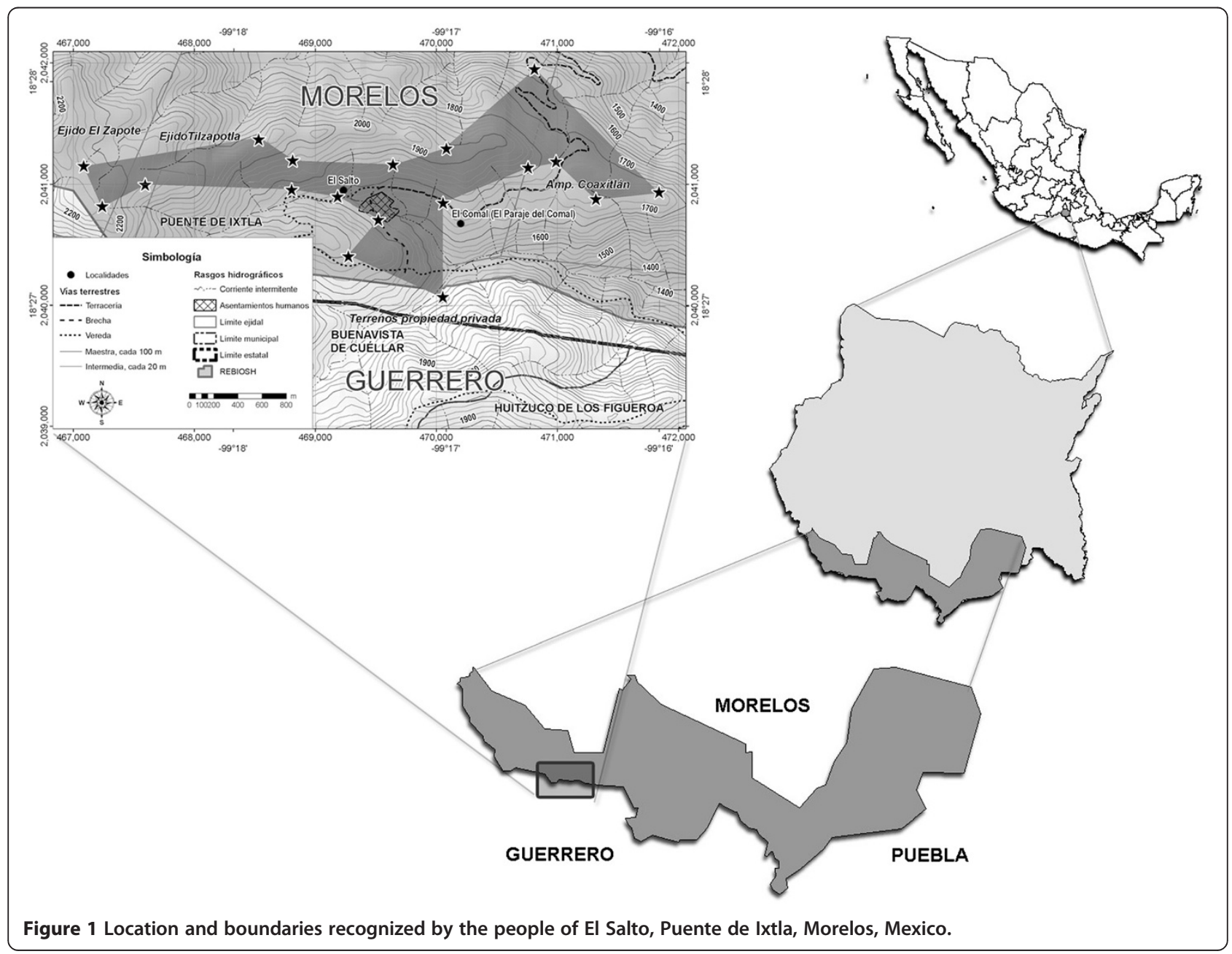


adults (60\%). Information of 41 inhabitants (21 women and 20 men), ranging between 30 and 70 years of age (average \pm SD: $51.4 \pm 16.3$ years), indicates that about $20 \%$ of the informants were born elsewhere, but they had lived at the locality for more than $40 \pm 8.6$ years. More than three fourths of the informants had finished primary education but $21.9 \%$ were illiterate. The festivities in the locality have a religious (mostly Catholic) and civic (school activities) character. The social organization is ruled by community assembly, whose highest authority is the Major's Assistant.

The main economic activity, practiced by $85.3 \%$ of the informants, was subsistence agriculture. Main crops include corn (Zea mays L.), bean (Phaseolus vulgaris L.) and squash (Cucurbita argyrosperma subsp. argyrosperma) in an integrated system. Subsistence agriculture was complemented by a range of activities related to the appropriation of natural resources, such as gathering and hunting (4.8\%), production of alcoholic beverages such as mezcal (made from Agave angustifolia Haw.) and "wine" (made from Vitis tiliifolia Humb. \& Bonpl. ex Roem. \& Schult.) (2.4\%) and of products derived from cattle, such as milk and cheese (9.7\%).

As much as $70.3 \%$ of the sample received economic support from the government, this support represented about $20 \%$ of their total income and therefore is an important complement to their main economic activities. The average monthly earnings per capita estimated for 2007 were \$USD $133.98 \pm 57.57$. Nevertheless, $44 \%$ of the people were exclusively living on remittances coming from the US. Rates of youth migration to the US, mainly after the young people have completed their highschool, are high.

\section{Ethnobotanical and socio-economic information}

Between September 2005 and December 2006, we conducted open-ended and semi-structured interviews $[45,46]$ with all the household heads $(n=41: 21$ women and 20 men). To avoid overestimating the knowledge about plants that might have been acquired elsewhere, we selected people older than 30 years old e.g., [47] who had been living in the study site for a minimum of 30 years $[48,49]$. Interviews were carried out individually to prevent distortions due to the presence of a third person $[48,49]$. The interviews focused on two aspects a) determining their knowledge of the plant resources (wild, cultivated and weedy species) in their community and b) characterizing the socio-economic conditions of the person being interviewed.

First, to quantify the degree of knowledge of plant resources and to identify the use value of each plant species, we conducted free listings by category of use [46]. The survey only included the theoretical dimension of ethnobotanical knowledge sensu [50]. Women's and men's knowledge of the plant richness of their community was defined as the number of species mentioned at the time of the interview. Second, we also asked about the following socio-economic information: 1) age, 2) gender, 3) education level (with or without primary studies); 4) origin (local or migrant), 5) main productive activities (farming or stockbreeding), 6) monthly expenditures, as a proxy for monetary earnings, 7) amount received in remittances, and 8) other economic activities (related to temporary earnings perceived as support from governmental institutional projects).

\section{Data analysis}

To determine the use-value of the local flora, we calculated the use-value (UV) index using the algorithm proposed by Phillips and Gentry [21,30], modified by Rossato et al. [51] and Lucena et al. [52]. The calculation was obtained by counting all the uses mentioned by every person for a specific plant and dividing the result by the total number of informants. The use-value corresponds to the average use associated to each species in a specific community:

$$
U V s=\Sigma U i s / n
$$

where $U_{i s}$ is the number of uses mentioned by an informant $i$, for each species $s$ and $n$ is the number of informants interviewed for each species.

We classified the local environment into five habitats: tropical deciduous forest, oak forest, riparian vegetation, home gardens and farming lands. The first three habitats correspond to vegetation types [43] and the other two to modified environments [53]. We visited each habitat repeatedly, with a different informant each time, to photograph and inventorying useful plants mentioned during the interviews and free listings. Subsequently, in a group meeting using an image projector, a photograph of each plant was showed to all interviewees to reach a consensus and verify that this was the correct etno-specie and avoid confusion by popular synonyms $[48,49]$. Then, after the identification of plant species was completed, we estimated the richness as the number of plant species per use category per habitat. The habitat of one species was determined based on Rzedowski [43]. The identification of botanical material was carried out at the HUMO Herbarium of the Universidad Autónoma del Estado de Morelos and with experts from the MEXU Herbarium of the Universidad Nacional Autónoma de Mexico. Voucher specimens were deposited at the HUMO and the folio number for each species is shown in (Table 1).

To determine the association of socio-economic factors and ethnobotanical knowledge, as measured in the described interviews, we used a non-metric multidimensional 
Table 1 Ethnofloral listing of El Salto

\begin{tabular}{|c|c|c|c|c|c|c|c|c|c|}
\hline Families & Scientific name & Common name & $\begin{array}{l}\text { Life } \\
\text { forms }\end{array}$ & $\begin{array}{c}\text { Uses } \\
\text { categories }\end{array}$ & $\begin{array}{c}\text { Vegetal } \\
\text { structure used }\end{array}$ & Habitat & Management & $\begin{array}{c}\text { Folio } \\
\text { number }\end{array}$ & $\begin{array}{l}\text { Use } \\
\text { value }\end{array}$ \\
\hline FABACEAE & Lysiloma acapulcense (Kunth) Benth. & Tepehuaje & A & $1,2,4,9,10$ y 12 & St, Co and Ro & Tdf & Wi & 26268 & 2,59 \\
\hline FAGACEAE & Quercus magnoliifolia Née & Encino amarillo & A & $1,9,10$ y 12 & St and Ro & Of & Wi & 26177 & 2,54 \\
\hline FABACEAE & Eysenhardtia polystachya (Ortega) Sarg. & Palo dulce o Coatle & A & $1,9,10$ y 12 & St, Co and Ro & Tdf & Wi & 26209 & 1,95 \\
\hline CUPRESSACEAE & Juniperus flaccida Schltdl. & Cedro & A & $2,4,8$ y 9 & St, Fr and Ro & Of & Wi & 26223 & 1,37 \\
\hline ROSACEAE & Rosa chinensis Jacq. & Rosa & Sh & 5 & $\mathrm{Fl}$ & $\mathrm{Hg}$ & $\mathrm{Gr}$ & 26286 & 1,37 \\
\hline MYRTACEAE & Psidium guajava $\mathrm{L}$. & Guayaba & A & 1,2 y 3 & $\begin{array}{l}\text { St, Lf, Co, Fr } \\
\text { and Ro }\end{array}$ & $\mathrm{Bg}$ & $\mathrm{Si}$ & 26187 & 1,29 \\
\hline FAGACEAE & Quercus castanea Née & Encino roble & A & $1,4,8,9,10$ y 12 & St, Co and Ro & Of & Wi & 26176 & 1,15 \\
\hline SAPINDACEAE & Ungnadia sp. & Asicincle & A & $2,4,9,10$ y 12 & St and Ro & Tdf & Wi & 26179 & 1,05 \\
\hline ORCHIDACEAE & Laelia autumnalis (La Llave \& Lex.) Lindl. & Catarina, Flor de San Diego & $\mathrm{He}$ & 7 y 11 & $\mathrm{Cp}$ and $\mathrm{Fl}$ & Of & Wi & 26161 & 1,02 \\
\hline CUCURBITACEAE & Cucurbita pepo L. & Calabaza & $\mathrm{He}$ & 3 & Fr and $\mathrm{Fl}$ & $\mathrm{Fl}$ & Gr & 26290 & 1,00 \\
\hline ERICACEAE & Arbutus xalapensis Kunth & Madroño & A & 3 y 10 & St, Ro and Fr & Of & Wi & 26110 & 1,00 \\
\hline FABACEAE & Mimosa lacerata Rose & Tecolhuixtle & Sh & 4,9 y 12 & St & Tdf & Wi & 26274 & 1,00 \\
\hline FABACEAE & Phaseolus vulgaris L. & Frijol & $\mathrm{He}$ & 3 & $\mathrm{Fr}$ & $\mathrm{Fl}$ & $\mathrm{Gr}$ & 26291 & 1,00 \\
\hline SOLANACEAE & Capsicum annuum L. & Chile & $\mathrm{He}$ & 3 & $\mathrm{Fr}$ & $\mathrm{Fl}$ & $\mathrm{Gr}$ & 26293 & 1,00 \\
\hline BURSERACEAE & Bursera fagaroides (Kunth) Engl. & Copal & A & 9 & St & Tdf & Wi & 26265 & 0,93 \\
\hline ASTERACEAE & Tagetes erecta $\mathrm{L}$. & Cempaxúchitl & $\mathrm{He}$ & 5 y 7 & $\mathrm{Cp}$ & $\mathrm{Hg}$ & $\mathrm{Gr}$ & 26259 & 0,88 \\
\hline SAPINDACEAE & Dodonaea viscosa Jacq. & Chapulixtle & A & $1,4,910$ y 12 & St, Lf and $\mathrm{Br}$ & Of & Wi & 26217 & 0,88 \\
\hline ASTERACEAE & Senecio salignus DC. & Jarilla & Sh & 7 & $\mathrm{Cp}$ & Of & Wi & 26246 & 0,85 \\
\hline FABACEAE & Harpalyce arborescens A. Gray. & Cahuira & A & $1,4,9$ y 12 & St, Lf and Co & Tdf & Wi & 26245 & 0,85 \\
\hline LAURACEAE & Nectandra globosa (Aubl.) Mez & Aile o Aguacatillo & A & $4,9,10$ y 12 & St and $\mathrm{Br}$ & Of & Wi & 26269 & 0,85 \\
\hline POACEAE & Zea mays $\mathrm{L}$. & Maíz & $\mathrm{He}$ & 1 y 3 & $\mathrm{Fr}$, Bra and In & $\mathrm{Fl}$ & $\mathrm{Gr}$ & 26292 & 0,85 \\
\hline RUTACEAE & Ruta chalepensis L. & Ruda & $\mathrm{He}$ & 1 y 7 & $\mathrm{Cp}$ & $\mathrm{Hg}$ & $\mathrm{Gr}$ & 26183 & 0,85 \\
\hline ASTERACEAE & Tagetes lunulata Ortega & Flor de muerto & $\mathrm{He}$ & 7 y 8 & $\mathrm{Cp}$ & Tdf & Wi & 26185 & 0,80 \\
\hline MALVACEAE & Hibiscus rosa-sinensis $\mathrm{L}$. & Tulipán & Sh & 5 & $\mathrm{Fl}$ & $\mathrm{Hu}$ & $\mathrm{Cu}$ & 26284 & 0,73 \\
\hline PIPERACEAE & Peperomia campylotropa A.W. Hill & Cilantro de peña & $\mathrm{He}$ & 3 y 11 & $\mathrm{Cp}$ & Of & Wi & 26231 & 0,73 \\
\hline FABACEAE & Senna skinneri (Benth.) H.S. Irwin \& Barneby & Paraca & A & 1,8 y 10 & $\begin{array}{l}\text { St, Fr, Co, Se } \\
\text { and Ro }\end{array}$ & Tdf & Wi & 26221 & 0,71 \\
\hline ANACARDIACEAE & Spondias mombin L. & Ciruela & A & 3 & $\mathrm{Fr}$ & $\mathrm{Hg}$ & Gr & 26113 & 0,68 \\
\hline FABACEAE & Conzattia multiflora (B.L. Rob.) Standl. & Guayacán & A & 1,9 y 12 & St and Se & Tdf & Wi & 26258 & 0,68 \\
\hline MALPIGHIACEAE & Malpighia mexicana A. Juss & Guajocote & Sh & 3 & Fr and $\mathrm{Co}$ & $\mathrm{Hg}$ & $\mathrm{Gr}$ & 26208 & 0,63 \\
\hline LAMIACEAE & Ocimum basilicum L. & Albahacar & $\mathrm{He}$ & 1 & $C p$ & $\mathrm{Hg}$ & $\mathrm{Gr}$ & 26182 & 0,61 \\
\hline ASTERACEAE & Artemisia ludoviciana Nutt. & Estafiate & $\mathrm{He}$ & 1 y 7 & $C p$ & $\mathrm{Hg}$ & $\mathrm{Gr}$ & 26181 & 0,59 \\
\hline
\end{tabular}


Table 1 Ethnofloral listing of El Salto (Continued)

\begin{tabular}{|c|c|c|c|c|c|c|c|c|c|}
\hline BALSAMINACEAE & Impatiens balsamina $\mathrm{L}$. & Chinos & $\mathrm{He}$ & 5 & $\mathrm{Fl}$ & $\mathrm{Hg}$ & Gr & 26278 & 0,56 \\
\hline FABACEAE & Leucaena macrophylla Benth. & Zacahuaje & A & 3 y 10 & St, Lf, Fl, Fr and Ro & Tdf & Gr & 26247 & 0,56 \\
\hline ASTERACEAE & Dahlia coccinea Cav. & Dalia de campo & Sh & 5 y 8 & $\mathrm{Fl}$ and $\mathrm{Ro}$ & Tdf & Wi & 26153 & 0,54 \\
\hline FAGACEAE & Quercus glaucoides M. Martens \& Galeotti & Chaparro & A & 8 y 10 & St, Lf, Fr and $\mathrm{Br}$ & Of & Wi & 26255 & 0,51 \\
\hline ASTERACEAE & Porophyllum macrocephalum DC. & Pápalos o Pepichas & $\mathrm{He}$ & 3 & $\mathrm{Cp}$ & Tdf & Wi & 26119 & 0,49 \\
\hline PORTULACACEAE & Portulaca oleracea L. & Verdolaga & $\mathrm{He}$ & 3 & $\mathrm{Cp}$ & Tdf & Wi & 26271 & 0,49 \\
\hline STERCULIACEAE & Guazuma ulmifolia Lam. & Cahuilote & A & $1,2,8,9,10$ y 12 & St, Fr, Lf and $\mathrm{Br}$ & Tdf & Wi & 26194 & 0,49 \\
\hline GERANIACEAE & Pelargonium domesticum L.H. Bailey & Geranio & $\mathrm{He}$ & 5 & $\mathrm{Fl}$ & $\mathrm{Hg}$ & Gr & 26279 & 0,46 \\
\hline VERBENACEAE & Vitex mollis H.B.K. & Cuayotomate & A & 1 y 8 & $\mathrm{Lf}, \mathrm{Fr}$ and $\mathrm{Co}_{0}$ & Tdf & Wi & 26244 & 0,39 \\
\hline APOCYNACEAE & Stemmadenia obovata K. Schum. & Tepechicle & A & 2 & $\mathrm{Br}$ & Tdf & Wi & 26211 & 0,37 \\
\hline APOCYNACEAE & Plumeria rubra L. & Rosal & A & 7 & $C p$ & Tdf & Wi & 26195 & 0,34 \\
\hline IRIDIACEAE & Gladiolus grandiflorus Andrews & Gladiola & $\mathrm{He}$ & 5 & $\mathrm{Fl}$ & $\mathrm{Hg}$ & Gr & 26282 & 0,29 \\
\hline POLEMONIACEAE & Loeselia mexicana (Lam.) Brand & Espinosilla & $\mathrm{He}$ & 1 & $C p$ & Of & Wi & 26165 & 0,27 \\
\hline FABACEAE & Acacia angustissima (Mill.) Kuntze & Timbre & A & 1 & $\mathrm{Br}$, $\mathrm{Co}$ and $\mathrm{Se}$ & Of & Wi & 26151 & 0,24 \\
\hline FABACEAE & Mimosa benthamii J.F. Macbr. & Tehuixtle & A & 4 y 9 & St & Tdf & Wi & 26210 & 0,24 \\
\hline AGAVACEAE & Agave inaequidens K. Koch & Maguey & $\mathrm{He}$ & 3 y 4 & In & Of & Wi & 26133 & 0,22 \\
\hline JUGLANDACEAE & Juglans regia $\mathrm{L}$. & Nogal & A & 3,4 y 9 & St and $\mathrm{Fr}$ & Gf & Wi & 26273 & 0,22 \\
\hline ULMACEAE & Celtis caudata Planch. & Estribillo & A & 12 & St & Tdf & Wi & 26159 & 0,22 \\
\hline ACANTHACEAE & Ruellia megasphaera Lindau & Té negro & $\mathrm{He}$ & 1 & Lf & $\mathrm{Hg}$ & Gr & 26239 & 0,20 \\
\hline ASTERACEAE & Matricaria recutita $\mathrm{L}$. & Manzanilla & $\mathrm{He}$ & 1 & $C p$ & $\mathrm{Hg}$ & Gr & 26285 & 0,20 \\
\hline MALPIGHIACEAE & Byrsonima crassifolia (L.) Kunth & Nanche & A & 1 y 3 & Fr and $\mathrm{Fl}$ & Tdf & Wi & 26275 & 0,20 \\
\hline BIGNONIACEAE & Tecoma stans (L.) Juss. ex Kunth & Iztamaxuchil o tronadora & A & 1 y 12 & St and Cp & Tdf & Wi & 26220 & 0,17 \\
\hline BURSERACEAE & Bursera ariensis (Kunth) McVaugh \& Rzed. & Cuajiote & A & 9 & St & Tdf & Wi & 26222 & 0,17 \\
\hline STERCULIACEAE & Waltheria americana $\mathrm{L}$. & Manrubio & $\mathrm{He}$ & 1 & $C p$ & Tdf & Wi & 26160 & 0,17 \\
\hline AMARANTHACEAE & Amaranthus hybridus L. & Quintonil & $\mathrm{He}$ & 3 & $\mathrm{Cp}$ & Tdf & Wi & 26146 & 0,15 \\
\hline CACTACEAE & Opuntia ficus-indica (L.) Mill. & Nopal & Sh & 1 y 3 & $\mathrm{Cl}$ and $\mathrm{Fr}$ & Tdf & Wi & 26240 & 0,15 \\
\hline LAMIACEAE & Salvia sessei Benth. & Vara de San Miguel & Sh & 10 & St and $\mathrm{Br}$ & Of & Wi & 26236 & 0,15 \\
\hline MELIACEAE & Swietenia humilis Zucc. & Palo quesero o Palo del zopilote & A & 1 & Co & Tdf & Wi & 26212 & 0,15 \\
\hline NYCTAGINACEAE & Bougainvillea glabra Choisy & Bugambilia & Sh & 5 & $\mathrm{Fl}$ & $\mathrm{Hg}$ & Gr & 26288 & 0,15 \\
\hline TAXODIACEAE & Taxodium mucronatum Ten. & Sabino & A & 4 & St & Gf & Wi & 26270 & 0,15 \\
\hline ANACARDIACEAE & Amphipterygium adstringens (Schltdl.) Standl. & Cuachalalate & A & 1 & Co & Tdf & Wi & 26180 & 0,12 \\
\hline ASTERACEAE & Viguiera sphaerocephala (DC.) Hemsl. & Ocotillo & Sh & 9,10 y 12 & St and $\mathrm{Br}$ & Of & Wi & 26117 & 0,12 \\
\hline ASTERACEAE & Tagetes lucida Cav. & Pericón & $\mathrm{He}$ & 1,7 y 11 & $\begin{array}{c}\mathrm{Cp}, \mathrm{Br}, \mathrm{Fl}, \mathrm{Co} \\
\text { and Se }\end{array}$ & Of & Wi & 26262 & 0,12 \\
\hline
\end{tabular}


Table 1 Ethnofloral listing of El Salto (Continued)

\begin{tabular}{|c|c|c|c|c|c|c|c|c|c|}
\hline FABACEAE & Leucaena esculenta (Moc. \& Sessé ex DC.) Benth. & Guaje colorado & A & 3 & $\mathrm{Fl}$ and $\mathrm{Fr}$ & $\mathrm{Hg}$ & Gr & 26219 & 0,12 \\
\hline LAMIACEAE & Salvia coccinea Buc'hoz ex Etl. & Mirto & $\mathrm{He}$ & 1 & Lf & $\mathrm{Hg}$ & $\mathrm{Gr}$ & 26287 & 0,12 \\
\hline ASTERACEAE & Vernonia alamanii DC. & Varaclacote & Sh & 12 & St & Tdf & Wi & 26131 & 0,10 \\
\hline ASTERACEAE & Montanoa arborescens DC. & Vara blanca & $\mathrm{He}$ & 5,7 y 9 & $\mathrm{Cp}, \mathrm{St}, \mathrm{Fl}$ and $\mathrm{Br}$ & Tdf & Wi & 26186 & 0,10 \\
\hline BURSERACEAE & Bursera bipinnata (DC.) Engl. & Copal chino & A & $1,2,4$ y 9 & $\mathrm{St}, \mathrm{Br}$ and $\mathrm{Lf}$ & Tdf & Wi & 26251 & 0,10 \\
\hline CHENOPODIACEAE & Teloxys ambrosioides (L.) W.A. Weber & Epazote & $\mathrm{He}$ & 3 y 7 & $C p$ & $\mathrm{Hg}$ & $\mathrm{Gr}$ & 26136 & 0,10 \\
\hline FABACEAE & Inga vera Willd. & Cajinicuil & A & 3 y 10 & $\mathrm{St}, \mathrm{Fr}$ and $\mathrm{Br}$ & Gf & Wi & 26152 & 0,10 \\
\hline SAPINDACEAE & Serjania triquetra Radlk. & Bejuco de tres costillas & $\mathrm{Cl}$ & 1 & $C p$ & Tdf & Wi & 26166 & 0,10 \\
\hline ANACARDIACEAE & Spondias purpurea L. & Ciruela de venado & A & 3 & $\mathrm{Fr}$ & Tdf & $\mathrm{Gr}$ & 26266 & 0,07 \\
\hline ASTERACEAE & Tagetes patula $\mathrm{L}$. & Flor de clemole & $\mathrm{He}$ & 5 y 7 & $\mathrm{Cp}$ and $\mathrm{Fl}$ & $\mathrm{Hg}$ & $\mathrm{Gr}$ & 26207 & 0,07 \\
\hline ASTERACEAE & Calea ternifolia Kunth var. ternifolia & Prodigiosa & $\mathrm{He}$ & 1 & $C p$ & Tdf & Wi & 26154 & 0,07 \\
\hline EUPHORBIACEAE & Euphorbia schlechtendalii Boiss. & Lechecillo & A & 1 & La & Of & Wi & 26193 & 0,07 \\
\hline FABACEAE & Erythrina breviflora Sessé \& Moc. ex DC. & Colorín & Sh & 3 & $\mathrm{Fl}$ & Tdf & Wi & 26191 & 0,07 \\
\hline LAMIACEAE & Salvia leucantha Cav. & Salvia & $\mathrm{He}$ & 7 & $\mathrm{Cp}$ & Of & Wi & 26254 & 0,07 \\
\hline LAURACEAE & Nectandra salicifolia (Kunth) Nees & Aguacachil & A & 1 & Lf and Co & Gf & Wi & 26163 & 0,07 \\
\hline RUBIACEAE & Randia tetracantha (Cav.) DC. & Caca de zorra o tecolosapo & A & 1 y 3 & Fr and $\mathrm{Co}$ & Tdf & Wi & 26197 & 0,07 \\
\hline ANACARDIACEAE & Comocladia engleriana Loes. & Teclate & A & 6 & $\mathrm{Cp}$ & Tdf & Wi & 26205 & 0,05 \\
\hline APOCYNACEAE & Mandevilla foliosa (Müll. Arg.) Hemsl. & Hierba de la cucaracha & $\mathrm{He}$ & 1 y 6 & $C p$ & Tdf & Wi & 26243 & 0,05 \\
\hline APOCYNACEAE & Cascabela thevetioides (Kunth) Lippold & Yoyote & A & 2,7 y 8 & Fr and St & Tdf & Wi & 26203 & 0,05 \\
\hline ARACEAE & Zantedeschia aethiopica (L.) Spreng. & Alcatraz & $\mathrm{He}$ & 5 & $\mathrm{Fl}$ & $\mathrm{Hg}$ & $\mathrm{Gr}$ & 26276 & 0,05 \\
\hline ASTERACEAE & Acourtia turbinata (Lex.) DC. & Cola de coyote & Sh & 1 & Lf & $\mathrm{Fl}$ & Wi & 26168 & 0,05 \\
\hline ASTERACEAE & Stevia connata Lag. & Pericón blanco & $\mathrm{He}$ & 2 y 7 & $\mathrm{Cp}$ and $\mathrm{Fl}$ & Of & Wi & 26169 & 0,05 \\
\hline ASTERACEAE & Artemisia absinthium L. & Ajenjo & $\mathrm{He}$ & 1 & $C p$ & $\mathrm{Hg}$ & Gr & 26277 & 0,05 \\
\hline ASTERACEAE & Bidens odorata Cav. & Mozote & $\mathrm{He}$ & 8 y 10 & $\mathrm{Cp}$ and $\mathrm{FI}$ & Tdf & Wi & 26138 & 0,05 \\
\hline BIGNONIACEAE & Crescentia alata Kunth & Cirian & A & 1 & $\mathrm{Fr}$ & Tdf & Wi & 26124 & 0,05 \\
\hline BOMBACACEAE & Pseudobombax ellipticum (Kunth) Dugand & Clavellina & A & 2 y 8 & St and $\mathrm{Fl}$ & Tdf & Wi & 26157 & 0,05 \\
\hline BORAGINACEAE & Cordia morelosana Standl. & Palo prieto & A & 1 & $\mathrm{Fl}$ & Tdf & Wi & 26263 & 0,05 \\
\hline BRASSICACEAE & Lepidium virginicum $\mathrm{L}$. & Mexixi & $\mathrm{He}$ & 1 & $C p$ & Tdf & Wi & 26162 & 0,05 \\
\hline BURSERACEAE & Bursera linanoe (La Llave) Rzed., Calderón \& Medina & Copal agüado & A & 2,8 y 9 & St and Lf & Tdf & Wi & 26242 & 0,05 \\
\hline CACTACEAE & Mammillaria nunezii (Britton \& Rose) Orcutt & Rodilla & $\mathrm{He}$ & 3 y 5 & $\mathrm{Fr}$ and $\mathrm{Fl}$ & Of & Wi & 26149 & 0,05 \\
\hline CAPPARIDACEAE & Cleome speciosa Raf. & Barbas de conejo & $\mathrm{He}$ & 5 & $\mathrm{Fl}$ & $\mathrm{Fl}$ & Wi & 26253 & 0,05 \\
\hline CARICACEAE & Carica papaya $\mathrm{L}$. & Papayo & A & 1 y 3 & Fr and $L f$ & $\mathrm{Hg}$ & $\mathrm{Gr}$ & 26283 & 0,05 \\
\hline CARICACEAE & Jacaratia mexicana A. DC. & Bonete & A & 3 & $\mathrm{Fr}$ and $\mathrm{Br}$ & Tdf & Wi & 26199 & 0,05 \\
\hline
\end{tabular}


Table 1 Ethnofloral listing of El Salto (Continued)

\begin{tabular}{|c|c|c|c|c|c|c|c|c|c|}
\hline CRASSULACEAE & Sedum corynephyllum Fröd. & Dedito de niño & $\mathrm{He}$ & 1 & Lf & $\mathrm{Hg}$ & $\mathrm{Gr}$ & 26280 & 0,05 \\
\hline EUPHORBIACEAE & Euphorbia fulva Staff & Pegahueso & A & 1 y 2 & $\mathrm{Br}$ and $\mathrm{La}$ & Tdf & Wi & 26218 & 0,05 \\
\hline EUPHORBIACEAE & Jatropha curcas L. & Mala mujer o Tepechicle & A & 1 y 3 & La & Of & Wi & 26190 & 0,05 \\
\hline FABACEAE & Acacia pennatula (Schltdl. \& Cham.) Benth. & Espino blanco & A & 8 & $\mathrm{Co}$ and $\mathrm{Fr}$ & Of & Wi & 26289 & 0,05 \\
\hline FABACEAE & Lysiloma divaricata Benth. & Mezquite & A & 9 y 10 & St and $\mathrm{Br}$ & Tdf & Wi & 26184 & 0,05 \\
\hline LAURACEAE & Litsea glaucescens Kunth & Laurel & $\mathrm{He}$ & 3 y 7 & $C p$ and $L f$ & $\mathrm{Hg}$ & Wi & 26272 & 0,05 \\
\hline LORANTHACEAE & Psittacanthus calyculatus (DC.) G. Don & Injerto de huizache & Sh & 1 & Lf & Tdf & Wi & 26196 & 0,05 \\
\hline MALVACEAE & Malva rotundifolia $\mathrm{L}$. & Malva & $\mathrm{He}$ & 1 y 3 & $\mathrm{Cp}$ & Tdf & Wi & 26147 & 0,05 \\
\hline OPILIACEAE & Agonandra racemosa (DC.) Standl. & Chicharroncillo & A & 1 & Lf & Tdf & Wi & 26216 & 0,05 \\
\hline OXALIDACEAE & Oxalis latifolia Kunth & Chucuyul & $\mathrm{He}$ & 3 & St & Of & Wi & 26230 & 0,05 \\
\hline ROSACEAE & Crataegus pubescens (C. Presl) C. Presl & Tejocote & A & 3 & $\mathrm{Fr}$ & Of & $\mathrm{Gr}$ & 26232 & 0,05 \\
\hline ROSACEAE & Eriobotrya japonica (Thunb.) Lindl. & Níspero & A & 1 y 3 & Fr and Lf & $\mathrm{Hg}$ & $\mathrm{Gr}$ & 26189 & 0,05 \\
\hline ROSACEAE & Rosa centifolia L. & Rosa de castilla & Sh & 1 & $\mathrm{Fl}$ & $\mathrm{Hg}$ & Wi & 26227 & 0,05 \\
\hline SOLANACEAE & Solanum lanceolatum Cav. & Sosa & Sh & 2 y 8 & $\mathrm{Lf}$ and $\mathrm{Cp}$ & Tdf & Wi & 26198 & 0,05 \\
\hline TILIACEAE & Heliocarpus terebinthinaceus (DC.) Hochr. & Cahuilahua & A & $1,4,8$ y 9 & St, Lf, Co and $\mathrm{Br}$ & Tdf & Wi & 26224 & 0,05 \\
\hline VERBENACEAE & Vitex hemsleyi Briq. & Querengue & A & 2,10 y 12 & St and $\mathrm{Br}$ & Tdf & Wi & 26248 & 0,05 \\
\hline VERBENACEAE & Lantana camara L. & Cinco negritos o Manzanito & Sh & 1 y 3 & Fr and $L f$ & Tdf & Wi & 26261 & 0,05 \\
\hline VITACEAE & Vitis tiliffolia Humb. \& Bonpl. ex Roem. \& Schult. & Bejuco de uva & $\mathrm{Cl}$ & 1,2 y 3 & $\mathrm{FI}$ and $\mathrm{Li}$ & Of & Wi & 26226 & 0,05 \\
\hline ACANTHACEAE & Justicia spicigera Schltdl. & Muicle & Sh & 1 & $\mathrm{Cp}$ & $\mathrm{Hg}$ & $\mathrm{Gr}$ & 26188 & 0,02 \\
\hline AGAVACEAE & Agave angustifolia Haw. & Agave de mezcal & $\mathrm{He}$ & 2 & $\ln$ & Of & Wi & 26249 & 0,02 \\
\hline AGAVACEAE & Agave horrida Lem. ex Jacobi & Agave de Ixtle & $\mathrm{He}$ & 2 & Bra & Of & Wi & 26150 & 0,02 \\
\hline AGAVACEAE & Polianthes geminiflora (Lex.) Rose & Aretito & $\mathrm{He}$ & 5 & $\mathrm{Fl}$ & Of & Wi & 26174 & 0,02 \\
\hline ANACARDIACEAE & Pseudosmodingium perniciosum (Kunth) Engl. & Cuajiote colorado & A & 6 & $\mathrm{Cp}$ & Tdf & Wi & 26225 & 0,02 \\
\hline APIACEAE & Eryngium columnare Hemsl. & Hierba del sapo & $\mathrm{He}$ & 1 & Lf & Of & Wi & 26229 & 0,02 \\
\hline ASCLEPIADACEAE & Asclepias glaucescens Kunth & Oreja de liebre & $\mathrm{He}$ & 1 & La & Tdf & Wi & 26252 & 0,02 \\
\hline ASCLEPIADACEAE & Marsdenia zimapanica Hemsl. & Pancololote & $\mathrm{Cl}$ & 3 & Fr and La & Tdf & Wi & 26241 & 0,02 \\
\hline ASPHODELACEAE & Aloe barbadensis Mill. & Sábila & $\mathrm{He}$ & 1 & $\mathrm{Fl}$ and $\mathrm{Lf}$ & $\mathrm{Hg}$ & Gr & 26202 & 0,02 \\
\hline ASTERACEAE & Sinclairia glabra (Hemsl.) Rydb. & Palo Santo o Campozano & A & 3 у 9 & $\mathrm{Fl}$, St and $\mathrm{Br}$ & Tdf & Wi & 26126 & 0,02 \\
\hline ASTERACEAE & Schkuhria pinnata (Lam.) Kuntze ex Thell. & Escobita & $\mathrm{He}$ & 2,4 y 10 & $\mathrm{Cp}$ & Tdf & Wi & 26139 & 0,02 \\
\hline ASTERACEAE & Pectis capillaris DC. & Limoncillo & $\mathrm{He}$ & 1 & $\mathrm{Cp}$ & Of & Wi & 26121 & 0,02 \\
\hline ASTERACEAE & Cosmos sulphureus Cav. & Flor amarilla & $\mathrm{He}$ & 3 & $\mathrm{Fl}$ & Tdf & Wi & 26235 & 0,02 \\
\hline ASTERACEAE & Senecio praecox (Cav.) DC. & Candelerillo & A & 5 & $\mathrm{Fl}$ & Tdf & Wi & 26250 & 0,02 \\
\hline ASTERACEAE & Calea urticifolia (Mill.) DC. & Canelilla & Sh & 1 & $\mathrm{Cp}$ & Tdf & Wi & 26167 & 0,02 \\
\hline
\end{tabular}


Table 1 Ethnofloral listing of El Salto (Continued)

\begin{tabular}{|c|c|c|c|c|c|c|c|c|c|}
\hline ASTERACEAE & Gnaphalium roseum Kunth & Gordolobo & $\mathrm{He}$ & 1 & $\mathrm{Cp}$ & Of & Wi & 26256 & 0,02 \\
\hline ASTERACEAE & Laennecia filaginoides DC. & Cimonilla & $\mathrm{He}$ & 1 & $C p$ & Tdf & Wi & 26118 & 0,02 \\
\hline ASTERACEAE & Psacalium megaphyllum (B.L. Rob. \& Greenm.) Rydb. & Churumbelo o Sombrerete & $\mathrm{He}$ & 1 & $\mathrm{Br}$ & Of & Wi & 26127 & 0,02 \\
\hline ASTERACEAE & Taraxacum officinale F.H. Wigg. & Hierba del golpe & $\mathrm{He}$ & 1 & $C p$ & $\mathrm{Fl}$ & Wi & 26172 & 0,02 \\
\hline ASTERACEAE & Adenophyllum porophyllum (Cav.) Hemsl. & Árnica & $\mathrm{He}$ & 1 & $\mathrm{Cp}$ & Tdf & Wi & 26115 & 0,02 \\
\hline ASTERACEAE & Tagetes micrantha Cav. & Anís & $\mathrm{He}$ & 3 & $C p$ & Of & Wi & 26123 & 0,02 \\
\hline ASTERACEAE & Tithonia tubiformis (Jacq.) Cass. & Acahual & $\mathrm{He}$ & 7 y 8 & $\mathrm{Cp}$ & $\mathrm{Fl}$ & Wi & 26140 & 0,02 \\
\hline ASTERACEAE & Verbesina crocata (Cav.) Less. & Capitaneja & $\mathrm{He}$ & 1 & Lf and St & Tdf & Wi & 26145 & 0,02 \\
\hline BEGONIACEAE & Begonia gracilis Kunth & Chucuyul de culebra & $\mathrm{He}$ & 7 & $C p$ & Of & Wi & 26137 & 0,02 \\
\hline BIGNONIACEAE & Jacaranda mimosifolia D. Don. & Jacaranda & A & 5 & $\mathrm{Fl}$ & $\mathrm{Hg}$ & $\mathrm{Gr}$ & 26215 & 0,02 \\
\hline BOMBACACEAE & Ceiba aesculifolia (Kunth) Britten \& Baker f. & Pochote & A & 1,8 y 12 & St, Fl and Sp & Tdf & Wi & 26143 & 0,02 \\
\hline BORAGINACEAE & Tournefortia hirsutissima $\mathrm{L}$. & Tlalchinol & $\mathrm{He}$ & 1 & $\mathrm{Cp}$ & $\mathrm{Fl}$ & Wi & 26132 & 0,02 \\
\hline BURSERACEAE & Bursera bicolor (Willd. ex Schltdl.) Engl. & Ticumaca & A & 1 y 4 & St and La & Tdf & Wi & 26214 & 0,02 \\
\hline BURSERACEAE & Bursera copallifera (DC.) Bullock & Copal tieso o Copal seco & A & $2,4,9$ y 12 & St & Tdf & Wi & 26264 & 0,02 \\
\hline BURSERACEAE & Bursera grandifolia (Schltdl.) Engl. & Palo mulato & A & 1 & Co & Tdf & Wi & 26200 & 0,02 \\
\hline CALOCHORTACEAE & Calochortus barbatus (Kunth) J.H. Painter & Campanita & $\mathrm{He}$ & 7 & $C p$ & Of & Wi & 26228 & 0,02 \\
\hline COCHLOSPERMACEAE & Cochlospermum vitifolium (Willd.) Spreng. & Panicua & A & 1 & St & Tdf & Wi & 26206 & 0,02 \\
\hline COMMELINACEAE & Tradescantia commelinoides Schult. \& Schult. f. & Lluvia & $\mathrm{He}$ & 1 & $\mathrm{Cp}$ & $\mathrm{Hg}$ & $\mathrm{Gr}$ & 26111 & 0,02 \\
\hline CONVOLVULACEAE & Ipomoea murucoides Roem. \& Schult. & Cazahuate prieto & A & $1,3,8,10$ y 11 & $\mathrm{St}, \mathrm{Fl}, \mathrm{Co}$ and $\mathrm{Br}$ & Tdf & Wi & 26141 & 0,02 \\
\hline CONVOLVULACEAE & Ipomoea pauciflora M. Martens \& Galeotti & Cazahuate blanco & A & 8 y 10 & $\mathrm{St}, \mathrm{Fl}$ and $\mathrm{Br}$ & Tdf & Wi & 26237 & 0,02 \\
\hline CONVOLVULACEAE & Ipomoea purpurea (L.) Roth & Quiebra plato & $\mathrm{Cl}$ & 5 & $\mathrm{Fl}$ & $\mathrm{Hg}$ & Wi & 26142 & 0,02 \\
\hline CRASSULACEAE & Kalanchoe pinnata (Lam.) Pers. & Orejona & $\mathrm{He}$ & 1 & Lf & $\mathrm{Hg}$ & $\mathrm{Gr}$ & 26164 & 0,02 \\
\hline CRASSULACEAE & Echeveria obtusifolia Rose & Siempreviva & $\mathrm{He}$ & 1 & $\mathrm{Fl}$ & Of & Wi & 26173 & 0,02 \\
\hline CRASSULACEAE & Sedum oxypetalum Kunth & Cuajiote de peña & Sh & 1 & Lf and St & Of & Wi & 26122 & 0,02 \\
\hline CUCURBITACEAE & Sechium edule (Jacq.) Sw. & Chayote & $\mathrm{Cl}$ & 1 y 3 & Fr and $L f$ & $\mathrm{Hg}$ & Gr & 26238 & 0,02 \\
\hline EUPHORBIACEAE & Euphorbia calyculata Kunth & Coralillo & A & 6 & $C p$ & Tdf & Wi & 26129 & 0,02 \\
\hline EUPHORBIACEAE & Ricinus communis L. & Higuerillo & $\mathrm{He}$ & 1 & Lf & $\mathrm{Fl}$ & Wi & 26109 & 0,02 \\
\hline FABACEAE & Canavalia villosa Benth. & Flor de gallito & $\mathrm{Cl}$ & 3 & $\mathrm{Fl}$ & Of & Wi & 26120 & 0,02 \\
\hline FABACEAE & Calliandra grandiflora (L'Hér.) Benth. & Cabellito de ángel & $\mathrm{He}$ & 1 y 3 & $\mathrm{Fl}$ and $\mathrm{Br}$ & Of & Wi & 26204 & 0,02 \\
\hline FABACEAE & Marina scopa Barneby & Escoba colorada & Sh & 4 & St & Tdf & Wi & 26135 & 0,02 \\
\hline FABACEAE & Phaseolus leptostachyus Benth. & Chinela & $\mathrm{He}$ & 3 & $\mathrm{Bu}$ & Tdf & Wi & 26171 & 0,02 \\
\hline FABACEAE & Senna hirsuta (L.) H.S. Irwin \& Barneby & Carnizuelo & $\mathrm{He}$ & 1 & Lf and $\mathrm{Fl}$ & Tdf & Wi & 26260 & 0,02 \\
\hline FABACEAE & Zornia thymifolia Kunth & Sangrinaria o Cascabelilllo & $\mathrm{He}$ & 1 & $\mathrm{Cp}$ & Of & Wi & 26155 & 0,02 \\
\hline
\end{tabular}


Table 1 Ethnofloral listing of El Salto (Continued)

\begin{tabular}{|c|c|c|c|c|c|c|c|c|c|}
\hline FABACEAE & Crotalaria cajanifolia Kunth & Crotalaria & $\mathrm{He}$ & 3 & $\mathrm{Fl}$ & Tdf & Wi & 26128 & 0,02 \\
\hline FABACEAE & Haematoxylum brasiletto H. Karst. & Palo de brasil & A & 1 & Co & Tdf & Wi & 26134 & 0,02 \\
\hline FABACEAE & Pithecellobium dulce (Roxb.) Benth. & Guamúchil & A & 3,9 y 10 & $\mathrm{St}, \mathrm{Fr}$ and $\mathrm{Br}$ & $\mathrm{Fl}$ & Wi & 26213 & 0,02 \\
\hline FLACOURTIACEAE & Xylosma flexuosa (Kunth) Hemsl. & Abrojo & A & 2 y 4 & St & Of & Wi & 26130 & 0,02 \\
\hline IRIDIACEAE & Tigridia multiflora (Baker) Ravenna & Gallito o Aretito & $\mathrm{He}$ & 7 & $\mathrm{Cp}$ & Of & Wi & 26233 & 0,02 \\
\hline LAMIACEAE & Salvia microphylla Kunth & Hierba del golpe & $\mathrm{He}$ & 1 & Lf & $\mathrm{Fl}$ & Wi & 26178 & 0,02 \\
\hline LAMIACEAE & Mentha piperita $\mathrm{L}$. & Hierbabuena & $\mathrm{He}$ & 1 y 3 & $\mathrm{Cp}$ & $\mathrm{Hg}$ & Gr & 26281 & 0,02 \\
\hline LILIACEAE & Bessera elegans Schult. f. & Aretito & $\mathrm{He}$ & 7 & $\mathrm{Cp}$ & Of & Wi & 26175 & 0,02 \\
\hline LOGANIACEAE & Buddleja americana $\mathrm{L}$. & Lengua de vaca & $\mathrm{He}$ & 1 & Lf & $\mathrm{Fl}$ & Wi & 26116 & 0,02 \\
\hline MALPIGHIACEAE & Galphimia glauca Cav. & Vara de San Agustín o Flor de Santa Teresa & Sh & 1 & $\mathrm{Cp}$ & Of & Wi & 26114 & 0,02 \\
\hline MALPIGHIACEAE & Bunchosia canescens (W.T. Aiton) DC. & Nanche de perro & Sh & 1 & $\mathrm{Cl}, \mathrm{Fl}$ and $\mathrm{Lf}$ & $\mathrm{Hg}$ & Gr & 26112 & 0,02 \\
\hline MORACEAE & Ficus cotinifolia Kunth & Cabrigo & A & 8 & $\mathrm{Fr}$ & Gf & Wi & 26267 & 0,02 \\
\hline ORCHIDACEAE & Stenorrhynchos lanceolatus (Audl.) Rich. & Espiguita & $\mathrm{He}$ & 5 & $\mathrm{Fl}$ & Of & Wi & 26144 & 0,02 \\
\hline PASSIFLORACEAE & Passiflora edulis Sims & Maracuya & $\mathrm{Cl}$ & 3 & $\mathrm{Fr}$ & $\mathrm{Hg}$ & Gr & 26148 & 0,02 \\
\hline PASSIFLORACEAE & Passiflora foetida $\mathrm{L}$. & Granada & $\mathrm{Cl}$ & 3 & $\mathrm{Fr}$ & Tdf & Wi & 26170 & 0,02 \\
\hline RUBIACEAE & Galium mexicanum Kunth & Pegarropa & $\mathrm{He}$ & 1 & $C p$ & Tdf & Wi & 26192 & 0,02 \\
\hline SCROPHULARIACEAE & Castilleja arvensis Schltdl. \& Cham. & Tornillo o cola de borrego & $\mathrm{He}$ & 1 & $\mathrm{Cp}$ & $\mathrm{Fl}$ & Wi & 26234 & 0,02 \\
\hline SELAGINELLACEAE & Selaginella lepidophylla (Hook. \& Grev.) Spring & Flor de piedra & $\mathrm{He}$ & 1 & $\mathrm{Cp}$ & Of & Wi & 26156 & 0,02 \\
\hline SOLANACEAE & Datura stramonium L. & Toloache & $\mathrm{He}$ & 1 y 7 & Lf and FI & $\mathrm{Fl}$ & Wi & 26257 & 0,02 \\
\hline SOLANACEAE & Nicotiana tabacum L. & Tenejiate & $\mathrm{He}$ & 2 & Lf & $\mathrm{Fl}$ & Wi & 26158 & 0,02 \\
\hline VERBENACEAE & Priva mexicana (L.) Pers. & Hierba del cáncer & $\mathrm{He}$ & 1 & $\mathrm{Cp}$ & Tdf & Wi & 26125 & 0,02 \\
\hline VERBENACEAE & Verbena carolina L. & Verbena & $\mathrm{He}$ & 1 & $\mathrm{Cp}$ & Of & Wi & 26201 & 0,02 \\
\hline
\end{tabular}

Life forms, A: Arboreal; Sh: Shrubby; He: Herbaceous; Cl: Climbing. Use categories, 1: Medicinal; 2: Crafts; 3: Edible; 4: Domestic wooden tools; 5: Ornamental; 6: Poison; 7: Mystical-religious; 8: Fodder; 9: Timber yieldingconstruction; 10: Firewood; 11: Commercialization of wild plants; 12: Farming wooden tools. Vegetal structure used: Fr: Fruit; Complete plant, Cp; Lf: Leaf; St: Stem; Br: Branch; In :Inflorescence; Fl: Flower; Bu: Bulb; Ro: Root; La: Latex; Bra: Bracts; Co: Cortex; Se: Seed; Sp: Spicules; Cl: Cladodium; Li: Liana. Habitat refers to the location where the species was collected and/or where local settlers gather it, Tdf: Tropical deciduous forest; Of: Oak forest; Hg: Home gardens; Fl: Farming lands; Gf: Gallery forest. Management, Wi: Wild; Gr: Grown. Use-value 0 "zero" corresponds specifically to species mentioned by local guides but not quantified in the interviews. 
scaling analysis (NMDS). This technique is appropriate for non-normal discontinuous data, such as the data used in this study. In our analysis, answers from the interviews were used as external variables to interpret ordination. Both the continuous variables (i.e., remittances, age, monthly expenditures), and the nominal variables, (i.e., main productive activities, education level, gender, origin, and other economic activities -earnings from a governmental source and origin of the person) were adjusted on the ordination. Two matrices were used to carry out the multivariate analysis. The main matrix included the useful species registered during open-ended and semi-structured interviews. The variables appear in rows and the interviewed people in columns. The secondary matrix included the socio-economic factors in rows and the interviewed people in columns.

The result from the analysis allowed for the representation of vectors as arrows that point in the direction towards which the variable being assessed changes the most; this is called direction of the gradient. The arrow's length is proportional to the correlation between the ordination and the assessed variable, and it is called force of the gradient. The analysis estimates a value for $r^{2}$, which represents the goodness of fit of the vector. Significance or p-value is based on the random permutations of the data. In the case of the factors, the analysis also estimates a value for $\mathrm{r}^{2}$ as goodness of fit, and a $\mathrm{p}$-value that allows to test the significance of the factor on the ordination. The graphic representation included the main groups' centroids. Only the vectors and factors that turned out to be significant $(\mathrm{p}<0.05)$ were graphically represented. The "Vegan" module [54] from the $\mathrm{R}$ program [55] was used for the statistical analyses. The difference in terms of the knowledge of the number of species between the genders was assessed for the six main use categories (Table 1).

Paired t-tests by household and a Wilcoxon signed rank test were used to determine if there were differences between men and women in terms of the number of species they knew by use category and in terms of the total number of species known. The paired difference distribution of paired t-test was analyzed by means of the Shapiro-Wilk's W normality tests.

\section{Results}

\section{Knowledge of plant species}

A total of 185 species, belonging to 149 genera and 69 families (Table 1), were recorded. We distributed those species in 12 use categories. We found a total of 310 different uses for the 185 species; thus, the richness of use is greater than the richness of species since some of the species had more than one use (Table 2). The richest families were Asteraceae and Fabaceae and the richest genera were Bursera and Tagetes.
According to their life form, the greatest proportion of useful plants registered at the locality included herbaceous (47\%), arboreal (38\%), shrubby (11\%) and climbing (4\%) species. The most used plant structures comprised the stem (28\%) and the complete plant (21\%), but there is no consistent pattern across the use categories (Table 1). The distribution of the richness of species use per habitat was consistent across habitat only in the case of the medicinal and edible species, the two most common uses. The ranking arrangement of the other use categories changed according to the habitat; this could be related to the availability of the species in each habitat (Table 2).

Tropical deciduous forest was the habitat with the highest proportion of useful species known to the sample, as it is shown by the greatest richness value of the useful plants and the greater diversity of uses for this habitat (Tables 2 and 3).

\section{Species use-value}

We defined three groups according to the species usevalues (Figure 2). The first group $(A)$ comprises three multi-purpose species (4 to 6 uses), wit use-values $>1.5$. Species in this group correspond to trees appreciated for their timber: tepehuaje (Lysiloma acapulcense (Kunth) Benth; 2,59), yellow oak (Quercus magnoliifolia Née; 2,54) and palo dulce (Eysenhardtia polystachya (Ortega) Sarg.; 1,95). The second group $(B)$ comprises 39 multipurpose species with use-values between 0.34 and 1.37 . Species in this group range from one (Rosa chinensis Jacq.; 1,37) to six uses (Quercus castanea Née; 1,15$)$, and many are used for medicinal purposes (11 species), timber (10 species), food (10 species), fuel (9 species), mystical-religious ceremonies (6 species), ornamental purposes ( 5 species), and commerce ( 2 species). The last group $(C)$ comprises 143 species with use-values between 0 and 0.29 , which show a distinct low diversity of uses per species. In fact this group includes only one taxon used for five purposes (Ipomoea murucoides Roem. \& Schult.; 0,02). Species in this group are mainly used for medicinal needs (76 species), timber (55 species), food (35 species), religious activities (15 species), ornamental and fuel (12 species), and commercial activities ( 2 species). Four species on the group are toxic.

\section{Socio-economic variables and their association to ethnobotanical knowledge}

Results from the NMDS analysis and subsequent adjustment of socio-economic variables suggested that the variables age, gender, farming and stockbreeding were associated in a statistically significant way to the knowledge of plant resources (Figures 3 and 4, Table 4). In contrasts, education level, origin, and the three others variables related to economic status (i.e., monetary 
Table 2 Use diversity among the 185 species and distribution in the five studied habitats

\begin{tabular}{|c|c|c|c|c|c|c|c|}
\hline Use categories & $\begin{array}{c}\text { Tropical deciduous } \\
\text { forest }\end{array}$ & $\begin{array}{c}\text { Oak } \\
\text { forest }\end{array}$ & $\begin{array}{c}\text { Riparian } \\
\text { vegetation }\end{array}$ & $\begin{array}{c}\text { Home } \\
\text { gardens }\end{array}$ & $\begin{array}{l}\text { Farming } \\
\text { lands }\end{array}$ & $\begin{array}{l}\text { Number of } \\
\text { species }^{1}\end{array}$ & Percentage \\
\hline Medicinal & 40 & 20 & 2 & 18 & 10 & 90 & 29 \\
\hline Edible & 19 & 11 & 3 & 10 & 4 & 48 & 15.5 \\
\hline $\begin{array}{l}\text { Timber yielding- } \\
\text { construction }\end{array}$ & 17 & 6 & 1 & 0 & 1 & 25 & 8.1 \\
\hline Firewood & 12 & 8 & 1 & 0 & 1 & 22 & 7.1 \\
\hline Crafts & 13 & 6 & 1 & 0 & 2 & 22 & 7.1 \\
\hline Mystical-religious & 4 & 9 & 0 & 6 & 2 & 21 & 6.8 \\
\hline Fodder & 14 & 3 & 1 & 0 & 1 & 19 & 6.1 \\
\hline Farming wooden tools & 13 & 5 & 0 & 0 & 0 & 18 & 5.8 \\
\hline Domestic wooden tools & 11 & 6 & 2 & 0 & 0 & 19 & 6.1 \\
\hline Ornamental & 3 & 3 & 0 & 11 & 1 & 18 & 5.8 \\
\hline Poison & 4 & 0 & 0 & 0 & 0 & 4 & 1.3 \\
\hline $\begin{array}{l}\text { Commercialization of } \\
\text { wild plants }\end{array}$ & 1 & 3 & 0 & 0 & 0 & 4 & 1.3 \\
\hline Total & 151 & 80 & 11 & 45 & 23 & 310 & 100 \\
\hline
\end{tabular}

Includes species with more than one use ( $n=310$ uses).

earnings, remittances, governmental monetary support) were not associated to the ethnobotanical knowledge of a person (Table 4 ).

The statistical analysis showed a clear spatial separation between two groups due to the differences in terms of men's and women's knowledge (Figure 3). Men from El Salto mentioned an average of $53.0 \pm 10$ (mean \pm standard deviation) plant species, whereas women referred to an average of $38.9 \pm 6$ plant species. The difference was statistically significant (Student's test, $\mathrm{T}_{15}=4.8$, $\mathrm{p}<0.001$ ). However, when analyzing the indication of plant species according to the general use category, we found that women significantly mentioned more ornamental and mystical-religious plants species than men (Table 5). In contrast, men mentioned significantly more plant species used for building houses or fences, crafts, farming wooden tools, firewood, domestic wooden tools, fodder, poisons, and the commercialization of wild plants than women (Table 5). No differences were found between men's and women's responses in relation to edible or medicinal plants (Table 5).

Table 3 Knowledge of plant species at natural and artificial environments in a mestizo community in the center of Mexico

\begin{tabular}{lcc}
\hline Habitat & Number of species & Percentage \\
\hline Tropical deciduous forest & 80 & 43.2 \\
Oak forest & 47 & 25.5 \\
Riparian vegetation & 6 & 3.2 \\
Home gardens & 35 & 18.9 \\
Farming lands & 17 & 9.2 \\
\hline
\end{tabular}

\section{Discussion}

\section{Ethnofloristic richness}

Species used at El Salto represent only $2.6 \%$ of the useful plants previously reported by Caballero and Cortés [9] for peasant -indigenous and mestizo- communities in Mexico. However, if we compare our data with the number of useful species reported by Bye et al. [56] for a larger territory comprising 12 ejidos near the region of Chamela, Jalisco, Mexico, the number of useful species

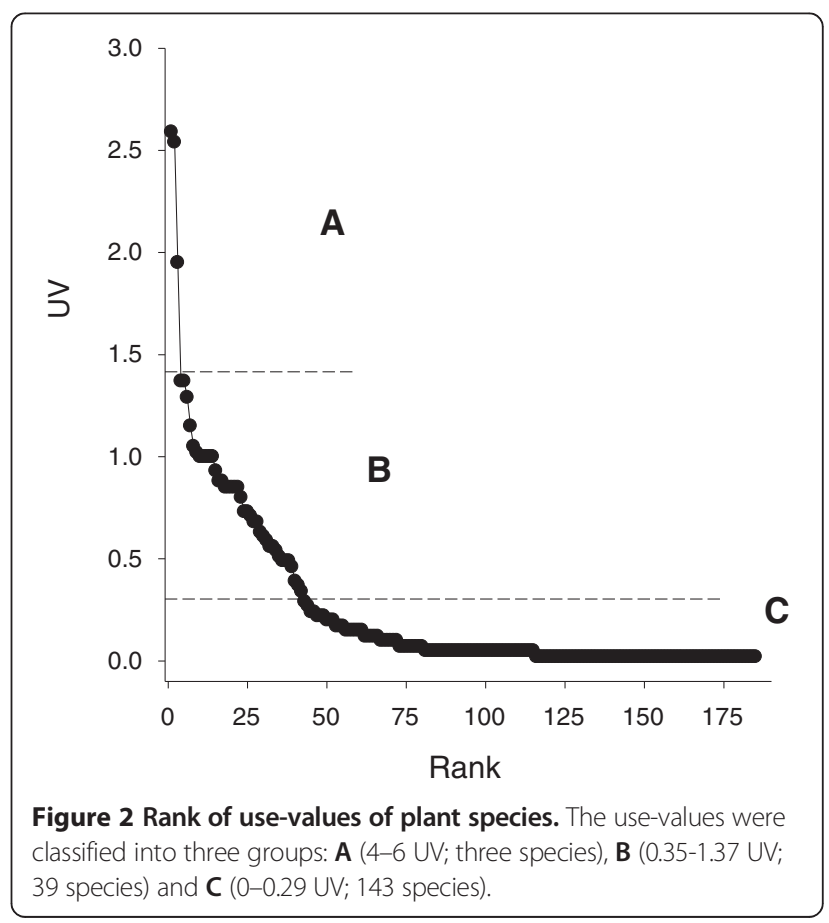




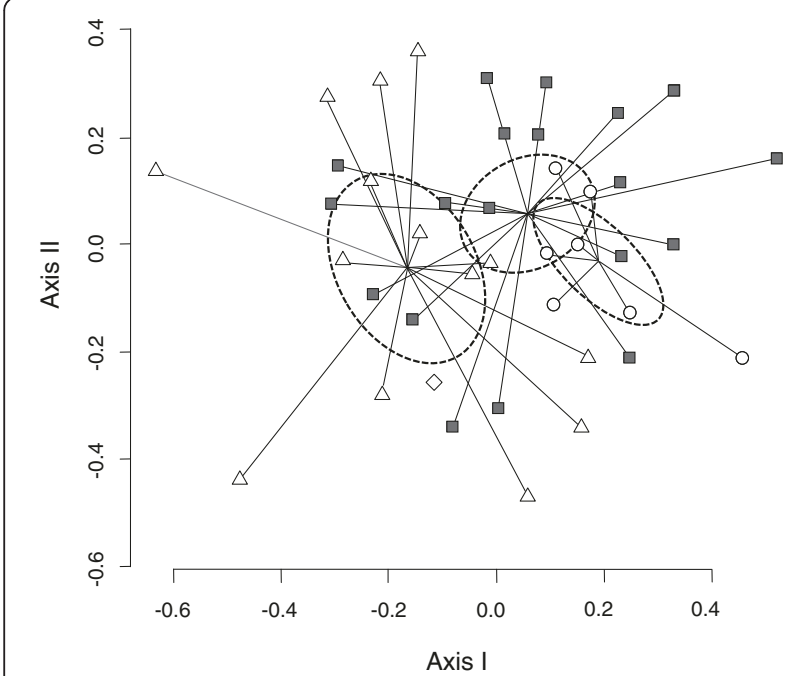

Figure 3 NMDS ordination of relationship between type of economic activity (stockbreeding, farming, both and others) and knowledge of plant species. The stockbreeding variable only includes one individual (diamond symbol). The farming group is represented by the set of grays-square symbols. Open circle symbols correspond to farming-stockbreeding practices. The remaining individuals (open triangle symbols) were grouped under others economic activity.

at El Salto is $12.4 \%$ higher than the number in those ejidos (185 vs. 162 species, respectively). This difference is more remarkable given that the ecological conditions are similar at both sites, suggesting that the size of the site is not the only factor that determines the number of useful species known by its inhabitants. Rather, historical, cultural and socioeconomic traits are essential to

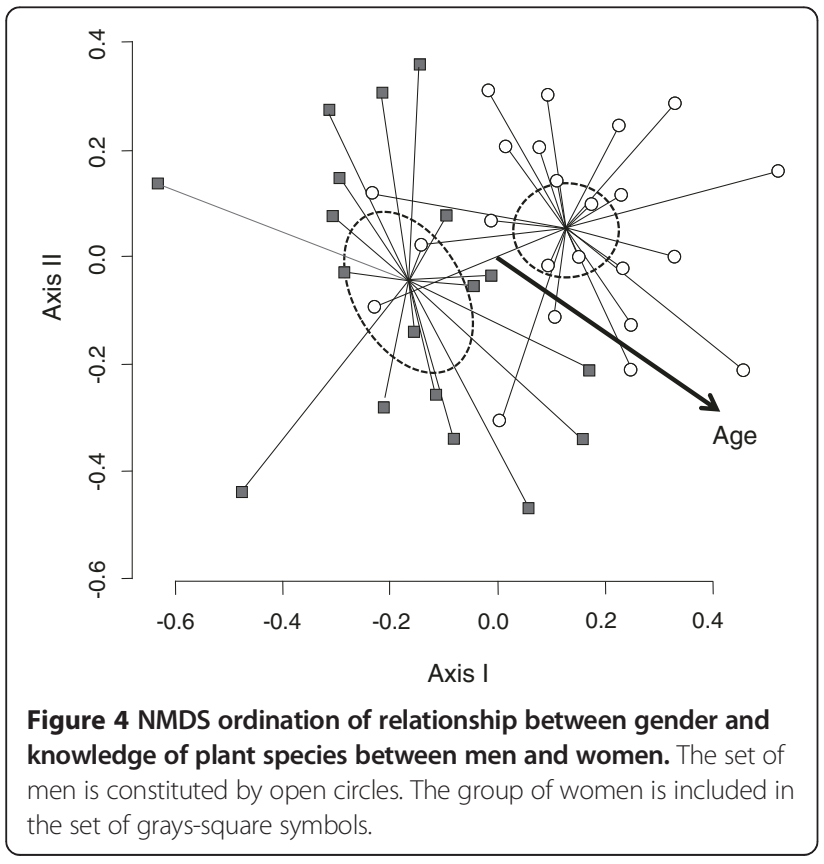

Table 4 Influence of socio-economic variables and factors on ethnobotanical knowledge at El Salto community

\begin{tabular}{lcc}
\hline Variables $^{\text {Pa }}$ & $\mathbf{P}^{\#}$ & $\mathbf{r}^{\mathbf{2}^{*}}$ \\
\hline Age $^{\mathrm{a}}$ & $\mathbf{0 . 0 2 0 4}$ & 0.18 \\
Gender $^{\mathrm{b}}$ & $<\mathbf{0 . 0 0 1}$ & 0.24 \\
Education level $^{\mathrm{b}}$ & 0.328 & 0.03 \\
Origin $^{\mathrm{b}}$ & 0.646 & 0.01 \\
Main productive activities $^{\mathrm{b}}$ & $\mathbf{0 . 0 0 6}$ & 0.20 \\
Monthly expenditures $^{\mathrm{a}}$ & 0.6986 & 0.02 \\
Remittances $^{\mathrm{a}}$ & 0.284 & 0.03 \\
Other economic activities $^{\mathrm{b}}$ & 0.177 & 0.04 \\
\hline
\end{tabular}

${ }^{\mathrm{a}}=$ Vectors; ${ }^{\mathrm{b}}=$ Factors; ${ }^{*}=$ P-values based on 1000 permutations (statistical significant $\mathrm{p}$-values are highlighted in bold); ${ }^{*}=$ Coefficient of determination.

understand the local knowledge of useful plant diversity in a particular study area [57].

The plants used at El Salto include some botanical families (Asteraceae, Fabaceae, Burseraceae, Lamiaceae, Verbenaceae, Euphorbiaceae, Anacardiaceae and Solanaceae) which play an important role to satisfy local needs, as well as some considered of uppermost importance at the national, state, and regional levels $[9,22,57]$. While these families contributed many useful species, the highest percentage of species listed came from a wide range of families, as follows: $7.3 \%$ of the families contributed three species each, $17.4 \%$ of the families contributed two species each, and $56.5 \%$ of the families contributed one species each. The high incidence of new uses of plants reported in the community could be accounted by the occurrence of many rare species ( $n=136$ species), which in turn could be explained by the presence of two major types of vegetation in the area: tropical deciduous and oak forests. In terms of the life forms of the resources, there is a high variation within each use category. Furthermore, our results indicate the preponderance of herbaceous species over other life forms, especially in modified environments (i.e., home gardens and farming lands); this fact is in agreement with the pattern reported for Mexico [9].

When analyzing the use value of plants, we find a predominance of medicinal uses. Moreover, this category is the one that displays more uses per species. This result appears to be constant among mestizo communities in the country, as it has been suggested by Bye [58] on a review of case studies among mestizo and indigenous groups in Mexico. Furthermore, this author has pointed out to differences in terms of the use of alimentary plants among the social groups mentioned: edible plants are of immediate concern for the indigenous societies while they only play a secondary role for mestizos.

We also found that people recognize a greater richness of species and diversity of uses in wild habitats than in modified environments. This finding contrasts with 
Table 5 Results of paired student $t$-test or Wilcoxon signed rank test ( $t$ and $W$, respectively) of plant species by use category mentioned by men and women at El Salto

\begin{tabular}{|c|c|c|c|c|}
\hline Use category & Number of species mentioned by men & Number of species mentioned by women & Test value & P-value \\
\hline Medicinal & $4.1 \pm 1.9$ & $4.7 \pm 1.9$ & $\mathrm{~T}=1.1$ & $P=0.31$ \\
\hline Edible & $5.3 \pm 1.1$ & $5.2 \pm 1.2$ & $W=22.5$ & $P=0.53$ \\
\hline Timber yielding construction & $7.5 \pm 1.4$ & $5.1 \pm 2.7$ & $\mathrm{~T}=2.9$ & $P=0.02$ \\
\hline Firewood & $3.1 \pm 0.9$ & $2.6 \pm 0.8$ & $W=32.5$ & $P=0.04$ \\
\hline Crafts & $3.5 \pm 1.9$ & $0.6 \pm 0.9$ & $W=120$ & $P<0.01$ \\
\hline Mystical-religious & $7.4 \pm 2.0$ & $8.8 \pm 1.9$ & $T=2.1$ & $P=0.049$ \\
\hline Fodder & $3.6 \pm 1.9$ & $0.7 \pm 1.1$ & $W=136$ & $P<0.01$ \\
\hline Farming wooden tools & $4.3 \pm 2.1$ & $1.6 \pm 1.8$ & $\mathrm{~T}=4.1$ & $P<0.01$ \\
\hline Domestic wooden tools & $4.0 \pm 2.2$ & $2.2 \pm 1.4$ & $T=2.7$ & $P=0.02$ \\
\hline Ornamental & $2.6 \pm 0.8$ & $3.2 \pm 0.5$ & $W=3$ & $P=0.04$ \\
\hline Poison & $3.8 \pm 1.0$ & $1.0 \pm 0.0$ & $W=136$ & $P<0.01$ \\
\hline Commercialization of wild plants & $3.8 \pm 0.9$ & $3.3 \pm 0.4$ & $W=21$ & $P=0.03$ \\
\hline
\end{tabular}

\#= Statistical significant p-values are highlighted in bold.

some studies that show that home gardens and farming lands harbor a greater biological diversity than the one registered in the wild environment [53,59]. Altieri et al. [53] show that tropical agroecosystems can contain more than 100 species; whereas Pulido et al. [59] point out that a family orchard with an average extension of 0.5-2.5 hectares located in the tropical deciduous forest holds a diversity comprising 92 species $(61 \%$ of them are native to the area). The differences could be methodological, as our study refers to species recognized, whereas the other studies are based on plant inventories. The differences could also be explained by the existence of a direct relation between the relative diversity of species in modified environments and the availability of irrigation water, as was described by Villa and Caballero [60]. Taking into account this relation, we hypothesize that the extended dry season that characterizes the region inhabited by the community of El Salto severely limits the amount of irrigation water, which in turn restricts the diversity of useful plant species that can be maintained in managed environments.

The results from this study reveal the importance that wild ecosystems have for mestizo communities in terms of the development of basic rural subsistence activities in dry tropical areas. However, our research also shows that both, wild habitats and artificial environments, are valuable to understand a group's ethnobotanical knowledge.

\section{Species use-values}

The highest use-values among species from groups $A$ and $B$ were registered in relation to timber, typically employed for house-building, firewood, and the manufacturing of farming tools, crafts, and household possessions. At present, these activities are not so frequent among the studied population, given the time and energy needed to manufacture products. However, even though these uses are less frequent, their knowledge persists, a situation that is similar to what Byg and Balslev [15] show for the use of palm species among the Shuar in Ecuador. For example, people maintain a body of knowledge related to timber species differentiating between spongy wood (hollow and brittle), solid wood (useful to manufacture farming tools), or wood that will become "good hot coal", i.e., firewood that lasts longer ignited [57].

When use-values were analyzed, we found that all of the species included in group $A$ (i.e., UV from 2.59 to 1.95) were mentioned by all the inhabitants and all of them were multi-purpose. To us, this finding illustrates the fact that the community has undergone a process of cultural appropriation of the floral diversity.

Even though groups $B$ (i.e., UV from 1.37 to 0.34 ) and $C$ (i.e., UV from 0.29 to 0.1 ) displayed the greatest floral diversity (182 species), there was a variation in terms of the multi-purposefulness and the number of uses was not stable. There was a tendency towards the use of timber yielding species in group $B$ and of medicinal species in group $C$, suggesting that group $B$ is probably the most important socially as it comprises the greatest number of species with the highest number of applications.

It is worth noting that none of the medicinal species displayed a high rate of use-value. In other words, the use of the most important plants for the community (i.e., the ones with highest use-value) is not related to the importance ranking by category of dominant use at the site. Such situation could be explained due to the multi-purposefulness that characterizes most species, since their inclusion in different use categories increases their potentiality, in other words, their use-value is 
enhanced while their exclusiveness for a specific use category decreases. Such speculation calls for a more complex analysis, since it is known that medicinal plants are culturally preeminent among mestizo communities [56].

\section{Variables associated to knowledge}

Our findings suggest that the knowledge of plant resources is associated mainly to socio-economic activities, age and gender, which is consistent with other ethnobotanical investigations $[6,12,15,25]$. In terms of the socio-economic aspects, despite the fact that occupation, i.e., farming and stockbreeding, was significantly associated to knowledge, it only accounted for $20 \%$ of the variation in the ethnobotanical knowledge. This suggests that there are other factors, not included in the analysis, which could influence ethnobotanical knowledge. Such other factors might entail cultural aspects such as ideological structures, ceremonies, significance and classification systems, production techniques and practices $[5,8,14,27,37]$, or ecological ones, which have historically been poorly explored -e.g., density of useful species, floral heterogeneity at the site, dominant biological forms, altitudinal variations, types of vegetation, selective floral and fruit morphology and phenology- [16,22,26,28,52,61,62].

Farming and stockbreeding constitute common activities that seem to provide a particular contribution to ethnobotanical knowledge. Some studies show that conducting primary activities contributes to use and management of natural resources [25,27]. The relation between animal rearing and ethnobotanical knowledge can be explained through several examples in this study. Thus, as livestock rearing constitutes the settlers' main activity, there is a need for them to know the plant resources that are helpful in the treatment of cattle's gastrointestinal diseases. This knowledge is based on the observation of the animals' alimentary habits with respect to wild and fodder plants, as well as on the detection of the toxic species that are eliminated from the environment to avoid that cattle consume them. Ethnobotanical knowledge also allows farmers to use alternative fodder in times of economic shortage or draught and also contributes to livestock health through the prevention of common diseases. Farmers are also familiar with the species that are used to craft farming tools, for house-building activities (with specific traits such as resistance, flexibility, duration and pliability, as living fences and as tutors). Some species are also tolerated due to the benefits they offer such as shade, medicine and food [57].

The informants' age also seem to be associated with ethnobotanical knowledge. Older people knew more useful plant species than younger people, probably because ethnobotanical knowledge tends to accumulate through the life cycle, as has been found elsewhere $[12,15,27,28]$.
Garro [63] indicates that aging is naturally associate with the process of knowledge acquisition as the pass of time help individuals accumulate knowledge and experience. Furthermore, in some studies, age seem to be the only variable associated with knowledge [64], although some other authors have found no association between age and knowledge [15]. Although most studies highlight knowledge differences between young and old people, our results suggest that ethnobotanical knowledge continues to accumulate after 30 years of age.

We also found differences between men's and women's knowledge in relation to the plants they use at the interspecific and the intracategorical levels, as has been pointed out by other authors e.g., [6,19,28]. Women's knowledge, in terms of the proportion of useful species they know, is closely associated to the treatment of diseases, the use of plants that embellish their household and of those related to rituals. The knowledge displayed by men is more diverse, since it includes a greater number of species used because of their wood quality to produce crafts and farming tools, to build houses, as fuel and household possessions, as well as species used as cattle fodder. Men are also more knowledgeable than women about plants that can cause bodily harm (swelling and irritation) during working days. Working with Raramuri indigenous people, Camou-Guerrero et al. [6] found that women had higher knowledge of medicinal and edible plants than men. As other authors [12], we did not find such differences in the community of El Salto. In sum, our results suggest that gendered division of labor within the family has resulted in constant interaction with the resources corresponding to specific activities. This phenomenon has determined how different species have acquired cultural importance for a specific gender in different cultural contexts $[2,65]$.

Other factor, such as the level of formal education, origin, and economic variables (income, remittances, and subsidies) are not associated to ethnobotanical knowledge. Previous research with young people has shown that the level of formal education bears a negative association with ethnobotanical knowledge [66], probably because time invested in schooling deflects from time invested in ethnobotanical knowledge also generating a lack of interest on the environment [26]. In contrast, Godoy [67] notes that formal education can lead to practices of use of more sustainable resources and the "environmental awareness". In most of the studies it has been found that education is associated with the loss of language and ethnobotanical knowledge in indigenous communities and of mixed origin (mestizo-indigenous) [27]. If the loss of indigenous language is the main factor that drives the loss of ethnobotanical knowledge, this could help explain why in the studied Spanish-speaking mestizo community we do not find the expected negative association between schooling and ethnobotanical knowledge. 
We did not find significant differences in ethnobotanical knowledge between people from the area and outsiders. The finding dovetails with what was reported by Byg and Balslev [15], who in their study in Ecuador find no differences between the ethnobotanical knowledge of indigenous peoples and colonists. In our case study, this could be due to the fact that people who have migrated to El Salto are coming from areas with the same type of vegetation and productive activities, and to the fact that most people migrated to the area during childhood.

Income, remittances and subsidies are all economic indicators of family well-being, which have also often been related to the loss of ethnobotanical knowledge, as income allows people to access market goods that substitute plant-made products $[15,34]$. However, we did not find such a relation. We argue that this could be due to the lack of large differences in the sample. In most cases families depend on remittances and subsidies, which are then invested in primary activities. Reyes-García et al. [68] show that conducting forest and farm activities is associated with greater ethnobotanical skills and with greater theoretical ethnobotanical knowledge, even if those are market oriented, thus implying that some forms of economic development can take place without eroding local ecological knowledge.

Although the variables presented have been analyzed independently, they do not act in independent way, or always have a linear relation with ethnobotanical knowledge. The acquisition of ethnobotanical knowledge is a complex process and we can not assure that the variables analyzed are the only direct drivers of the transmission and acquisition of this knowledge. We suggest that the generation of ethnobotanical knowledge should be understood as a dynamic social process, driven by the current interaction with the ecosystem given the importance of multiple socioeconomic and cultural factors [69].

\section{Conclusions}

We found that the ethnobotanical knowledge of a mestizo community settled in a tropical deciduous forest environment is actually larger than the ethnobotanical knowledge reported for other regions in the same environment, which is widely documented in Mexican literature. Thus, it is considered that ethnobotanical research among mestizo populations is essential to detect locations with vegetal and cultural richness in order to build up the implementation of interdisciplinary programs that favor the development of feasible local proposals for biocultural conservation, particularly in cultural strengthening of traditional knowledge systems for an effective forest management.

The use-value rate constitutes a useful tool to approach a group's socio-economic and cultural expressions, since it allows the most used species as well as tendencies in use. Nevertheless, it is also necessary to assess the species' frequency of use, since although species might be known and valued, they might not be currently in use. The use-value rate also allows to assess directly the pressure being exerted on a particular species or on a vegetal community.

While the use-value technique proposed by Phillips and Gentry [70] has limitations related to the interpretation of the pressure to use vegetation resources, it is important to note that the use of any natural resource is performed within specific cultural contexts [14,26,62,71]. Studies such as this one, with a focus on the relation between the local lore and native plants, can become important tools for the conservation of tropical resources by establishing management strategies based on local demands and by prioritizing the selection of species in terms of conservation efforts. Therefore, as has been mentioned by Lawrence et al. [19], a key challenge for ethnobotanists is to develop effective ways of understanding both people and the value of plants and more particularly of revealing the socio-economic context and the ecological values that influence them.

The knowledge produced during the interaction between plants and peasant societies is diverse and selective, in other words, the wisdom that articulates the use of plant resources varies according to the type of vegetation present in the location, the cultural value of certain plants as well as their economic and social relevance. In this study we found that socio-economic variables, such as those related both to farming/stockbreeding activities and to the differences according to age and gender, have a strong association to the ethnobotanical knowledge. Older people reported more useful plant species and could be an important cultural reservoir of ethnobotanical knowledge in mestizo rural communities. Men's and women's ethnobotanical knowledge differed in terms of use categories and of the number of species they recognize; this is expressed by different patterns of cultural appropriation and reproduction concerning the use of certain species. Nevertheless, there is consensus related to the knowledge of medicinal and alimentary plants at the family and community levels.

The use of this type of information could be very valuable for studies directed towards the restoration of ecosystems with species of local importance, particularly if the most valued plant resources known to men and women are taken into account. Identifying these resources could enhance the chances of success as well as the sustainability of silvicultural programs oriented to biological conservation and rural development.

Finally, ethnobotanical knowledge, understood as a dynamic and socially specific process, deserves deeper study to determine its origin, transformation and its possible loss. This will allow assessment and systematization 
of patterns of plant knowledge, which involves possibilities of political actions in programs to strengthen knowledge and the sociocultural, economic and ecological factors that are related to prevent their erosion. The results of this study contribute to integrate the local knowledge of a mestizo community into appropriate proposals to preserve it.

\section{Consent}

Prior informed consent was orally obtained from all participants, in line with the requirements of the Universidad Autónoma del Estado de Morelos ethical prescriptions for publication of this report and any accompanying images.

\section{Competing interests}

The authors articulate that they have no competing interest.

\section{Authors' contributions}

LB main author, involved in the study design, conducting of interview, field work, literature review and general data collection and systematization, wrote the first draft and concluded the final version of this paper with the rest of the coauthors. $\mathrm{AO}$ and $\mathrm{BM}$ main coordinators of the research project; contributed with original ideas and data, and participated in reviewed several drafts of the manuscript. NM suggested and performed statistical analysis, teaming with $\angle B, A O$ and $B M$ in the review of drafts and in the final version this paper. VR contributed to review drafts of the paper and the final manuscript. All authors read and approved the final manuscript.

\section{Authors' information}

LB student at the Postgraduate of Botany, Colegio de Postgraduados. AO, NM and BM full time researchers at CIBYC, UAEM. VR researcher at the Universitat Autónoma de Barcelona, Spain.

\section{Acknowledgements}

We thank the financial support of the Center for Biodiversity Research and Conservation of Morelos State University for the development of this research. Thanks also the curator of the Herbarium "HUMO" Morelos state for the support for taxonomic identification of some species. The authors thank specially to people of the El Salto for their hospitality, generosity and participation in interviews and sharing their knowledge.

\section{Author details \\ ${ }^{1}$ Facultad de Ciencias Biológicas, Universidad Autónoma del Estado de Morelos, Av. Universidad No 1001, Cuernavaca CP: 62210, Mexico. ${ }^{2}$ Present address: Postgrado en Botánica, Colegio de Postgraduados, Montecillo, Texcoco C.P. 56230, México. ${ }^{3}$ Centro de Investigación en Biodiversidad y Conservación (CIByC), Av. Universidad No 1001, Cuernavaca CP: 62210, Mexico. ${ }^{4}$ ICREA and Institut de Ciencia i Tecnologia Ambientals, Universitat Autónoma de Barcelona, 08193 Bellatera, Barcelona, Spain.}

Received: 13 July 2013 Accepted: 15 January 2014 Published: 27 January 2014

\section{References}

1. Berkes F: Sacred ecology: Traditional ecological knowledge and resource management. United States of America: Taylor \& Francis Press; 1999.

2. Hassanein N: Changing the way America farms: knowledge and community in the sustainable agriculture movement. United States of America: University of Nebraska Press; 1999

3. Casas A, Otero-Arnaiz A, Pérez-Negrón E, Valiente-Banuet A: In situ management and domestication of plants in Mesoamerica. Ann Bot 2007, 100:1101-1115.

4. Hernández XE: Aspects of plant domestication in Mexico: a personal view. In Biological diversity of Mexico: origins and distribution. Edited by Ramamoorthy T, Bye R, Lot A, Fa J. New York: Oxford Univ; 1993:733-753.
5. Ross N, Barrientos T, Esquit-Choy A: Triad tasks, a multipurpose tool to elicit similarity judgments: the case of Tzotzil Maya plant taxonomy. Field Methods 2005, 17:269-282.

6. Camou-Guerrero A, Reyes-García V, Martínez-Ramos M, Casas A: Knowledge and use value of plant species in a Rarámuri community: a gender perspective for conservation. Hum Ecol 2008, 36:259-272.

7. Benz B, Cevallos J, Santana F, Rosales J, Graf S: Losing knowledge about plant use in the Sierra de Manantlán Biosphere Reserve, Mexico. Econ Bot 2000, 54:183-191.

8. Ross N: Lacandon Maya intergenerational change and the erosion of folk biological knowledge. In Ethnobiology and biocultural diversity. Edited by Stepp J, Wyndham F, Zarger R. Athens: University of Georgia; 2002:585-592.

9. Caballero J, Cortés L: Percepción, uso y manejo tradicional de los recursos vegetales en México. In Plantas, cultura y sociedad: estudio sobre la relación entre seres humanos y plantas en los albores del siglo XXI. Edited by Rendón B, Rebollar S, Caballero J, Martínez M. México: UAM-Ixtapalapa-SEMARNAT; 2001:79-100.

10. Toledo V, Ortiz-Espejel B, Cortéz L, Moguel P, Ordoñez de Jesús M: The multiple use of tropical forests by indigenous peoples in Mexico: a case of adaptative management. Conserv Ecol 2003, 7:9.

11. Argueta A: Atlas de las plantas de la medicina tradicional mexicana. Mexico: Instituto Nacional Indigenista Press; 1994.

12. Ryan C, Guido P, Doel S: Factors in maintaining indigenous knowledge among ethnic communities of Manus island. Econ Bot 2005, 59:356-365.

13. Wekerle $C$, Huber $F$, Yongping $Y$, Weibang S: Plant knowledge of the Shuhi in the Hengduan Mountains, southwest China. Econ Bot 2006, 60:3-23.

14. Turner N, Ari Y, Berkes F, Davidson-Hunt I, Fusun Z, Miller A: Cultural management of living trees: an international perspective. J Ethnobiol 2009, 29(2):237-270

15. Byg A, Balslev $\mathrm{H}$ : Factors affecting local knowledge of palms in Nangaritza Valley in southeastern Ecuador. J Ethnobiol 2004, 24:255-278,

16. Akerreta S, Cavero R, López V, Calvo M: Analyzing factors that influence the folk use and phytonomy of 18 medicinal plants in Navarra. J Ethnobiol Ethnomed 2007, 3:16.

17. Lepofsky C: The past, present, and future of traditional resource and environmental management. J Ethnobio/ 2009, 29(2):161-166.

18. Benz B, Santana F, Pineda R, Cevallos J, Robles L, Deniz D: Characterization of mestizo plant use in the Sierra de Manantlán, Jalisco-Colima, México. J Ethnobiol 1994, 14:123-141.

19. Lawrence A, Phillips O, Reategui A, Lopez M, Rose S, Wood D, Farfan A: Local values for harvester forest plants in Madre de Dios Peru: towards a more contextualised interpretation of quantitative ethnobotanical data. Biodivers Conserv 2005, 14:45-79.

20. Merino-Pérez: Las condiciones de las comunidades forestales mexicanas y la política pública. Recuento de desencuentros. In La Naturaleza en contexto: Hacia una ecología política mexicana. 1st edition. Edited by Durand L, Figueroa F, Guzmán M. México, D.F: UNAM, Centro de Investigaciones Interdisciplinarias en Ciencias y Humanidades; 2012:33-63.

21. Castillo A, Magaña A, Pujadas A, Martínez L, Godínez C: Understanding the interaction of rural people with ecosystems: a case study in a tropical Dry forest of Mexico. Ecosystems 2005, 8:630-643.

22. Maldonado B, Caballero J, Delgado-Salinas A, Lira R: Relationship between Use value and ecological importance of floristic resources of seasonally Dry tropical forest in the Balsas river basin, Mexico. Econ Bot 2013, 67:17-29.

23. Caballero J, Casas A, Cortés L, Mapes C: Patrones en el conocimiento, uso y manejo de las plantas en pueblos indígenas de México. Estudios Atacameños 1998, 16:181-196.

24. Hersch-Martínez P, González L: Investigación participativa en etnobotánica. Algunos procedimientos coadyuvantes en ella. Dimens Antropol 1996, 3:129.

25. Martínez-Ballesté A, Martorell C, Caballero J: Cultural or ecological sustainability? The effect of cultural change on Sabal palm management among the lowland Maya of Mexico. Ecol Soc 2006, 11(2):27.

26. Blancas J, Casas A, Pérez-Salicrup D, Caballero J, Vega E: Ecological and socio-cultural factors influencing plant management in Náhuatl communities of the Tehuacán Valley, Mexico. J Ethnobiol Ethnomed 2013, 9:39.

27. Saynes-Vásquez A, Caballero J, Meave y J, Chiang F: Cultural change and loss of ethnoecological knowledge among the Isthmus Zapotecs of Mexico. J Ethnobiol Ethnomed 2013, 9:40.

28. Phillips O, Gentry A: The useful plants of Tambopata, Perú I: statistical hypotheses tests with a new quantitative technique. Econ Bot 1993, 7:15-32.

29. Hanazaki N, Tamashiro J, Leitão-Filho H, Begossi A: Diversity of plant uses in two Caiçara communities from the Atlantic Forest coast, Brazil. Biodivers Conserv 2000, 9:597-615. 
30. Voeks R, Leony A: Forgetting the forest: assessing medicinal plant erosion in Eastern Brazil. Econ Bot 2004, 58:294-306.

31. Kristensen $M$, Balslev $H$ : Perceptions, use and availability of woody plants among the Gourounsi in Burkina Faso. Biodivers Conserv 2003, 12:1715-1739.

32. Dufour D: Use of tropical rainforests by native Amazonians. Bioscience 1990, 40:652-659.

33. Pichon F: Land-use strategies in the Amazon Frontier: farm-level evidence from Ecuador. Hum Organ 1996, 55:416-424.

34. Holmes C: Assessing the perceived utility of wood resources in a protected area of western Tanzania. Conserv Biol 2003, 111:179-189.

35. Varughese $\mathrm{G}$, Ostrom $\mathrm{E}$ : The contested role of heterogeneity in collective action: some evidence from community forestry in Nepal. World Dev 2001, 29:747-765.

36. Jackson C: Gender Analysis and Environmentalism. In Social Theory and the Global Environment. Edited by Redclift M, Benton T. New York: Routledge; 1994:113-149.

37. Zent S: Acculturation and ethnobotanical knowledge loss among the Piaroa of Venezuela: demonstration of a quantitative method for the empirical study of traditional ecological knowledge change. In On Biocultural Diversity: Linking Language, Knowledge, and the Environment. Edited by Maffi L. Washington DC: Smithsonian Institution; 2001:190-211.

38. Otero-Rozas E, Ontillera-Sánchez R, Sanosa P, Gómez-Baggethun E, Reyes-García V, González J: Traditional ecological knowledge among transhumant pastoralists in Mediterranean Spain. Ecol Soc 2013, 18(8):33.

39. Convention on biological diversity. http://www.cbd.int/doc/legal/cbd-en.pdf.

40. LEGEEPA (Ley general del equilibrio ecológico y protección al ambiente). http:// biblioteca.semarnat.gob.mx/janium/Documentos/Ciga/agenda/DOFsr/148.pdf.

41. Nabhan G: Singing the turtles to sea. The Comcaác (seri) art and science of reptiles. United States of America: University of California Press; 2003.

42. INEGI: Síntesis geográfica del estado de Morelos. Secretaría de Programación y Presupuesto. México: Instituto Nacional de Estadística y Geografía Press; 1981.

43. Rzedowski J: Vegetación de México. Mexico: Limusa Press; 1978.

44. Taboada M, Granjeno A, Guadarrama O: Normales climatológicas (temperatura y precipitación) del estado de Morelos. México: Universidad Autónoma del Estado de Morelos Press; 2007.

45. Bernard R: Estructured interviewing. In Research methods in cultural anthropology: qualitative and quantitative approaches. 2nd edition. Edited by Bernard R. London: Altamira Press; 1994:237-255.

46. Martin G: Ethnobotany: A methods manual. Cambridge: Chapman \& Hall Press; 1995.

47. Begossi A, Hanazaki N, Tamashiro J: Medicinal plants in the Atlantic forest (Brazil): knowledge, use and conservation. Hum Ecol 2002, 30:281-299.

48. Albuquerque $P$, Lucena R: Métodos e técnicas para a coleta de dados. In Métodos e técnicas na pesquisa etnobotânica. Edited by Albuquerque $\mathrm{P}$, Lucena R. Recife, Brasil: NUPEEA; 2004

49. Monteiro JM, Lucena RFP, Alencar NL, Nascimento VT, Araújo TAS, Albuquerque UP: When intention matters: comparing three ethnobotanical data collection strategies. In Current topics in ethnobotany. Edited by Albuquerque UP, Ramos MA. Kerala, India: Research Signpost; 2008:113-124

50. Reyes-García V, Martí N, Mcdade T, Tanner S, Vadez V: Concepts and methods in studies measuring individual ethnobotanical knowledge. J Ethnobiol 2007, 27:182-203.

51. Rossato SC, Leitáo-Filho HF, Begossi A: Ethnobotany of Caicaras of the Atlantic forest coast (Brazil). Econ Bot 1999, 53:387-395.

52. Lucena R, Lima $E$, Albuquerque U: Does the local availability of woody Caatinga plants (northeastern Brazil) explain their use value? Econ Bo 2007, 61:347-361.

53. Altieri M, Anderson $K$, Merrick L: Peasant agriculture and the conservation of crop and wild plant resources. Conserv Biol 1987, 1:49-58.

54. Oksannen J, Kindt R, Legendre P, O'Hara B: Vegan: community ecology package version 1.8-5. http://cran.r-project.org/.

55. R Development Core Team: A language and environment for statistical computing. Vienna, Austria: R Foundation for Statistical Computing. ISBN 3-900051-07-0. URL [http://www.R-project.org]

56. Bye $R$, Cervantes $L$, Rendón B: Etnobotánica en la región de Chamela, Jalisco, México. In Historia natural de Chamela. Edited by Noguera F, Vega J, García A, Quesada M. México: Instituto de Biología, UNAM; 2002:545-559.

57. Monroy-Ortiz C, Monroy R: Análisis preliminar de la dominancia cultural de las plantas útiles en el estado de Morelos. Bol Soc Bot México 2004, 74:77-95.
58. Bye R: Ethnobotany of the Mexican tropical dry forest. In Seasonally dry tropical forest. Edited by Bullock SH, Mooney HA, Medina E. UK: Cambridge University; 1995:423-438.

59. Pulido T, Pagaza-Calderón E, Martínez-Ballesté A, Maldonado-Almanza B, Saynes A, Pacheco R: Home gardens as an alternative for sustainability: challenges and perspectives in Latin America. In Current topics in ethnobotany. Edited by Albuquerque UP, Ramos MA. Kerala, India: Research Signpost; 2008:55-79.

60. Villa A, Caballero J: Floristic variation in Nahua homegardens of Guerrero, Mexico. Abstracts of Presentations at the 18th annual conference of the Society of Ethnobiology. J Ethnobiol 1995, 15(2):287-310. Tucson, Arizona.

61. Guèze M, Luz A, Paneque-Gálvez J, Macia M, Orta-Martínez M, Pino J, Reyes-García V: Are ecologically important tree species the most useful? A case study from indigenous people in the Bolivian Amazon. Economic Botany 2014. 10.1007/s12231-014-9257-8.

62. Albuquerque UP, Lucena RFP: Can apparency affect the use of plants by local people in tropical forest? Interciencia 2005, 30:506-511.

63. Garro LC: Intracultural variation in folk medical knowledge: a comparison between curers and noncurers. American Anthropological 1986, 88:351-370.

64. Aguilar S: Etnobotánica cuantitativa en una región de Bosque de Niebla de Sierra Norte, Oaxaca. MSc thesis, Instituto Politécnico Nacional; 2007.

65. Howard P: Gender and social dynamics in swidden and homegardens in Latin America. In Tropical homegardens. A time-tested example of sustainable agroforestry. Edited by Kumar BM, Nair PKR. Dordrecht: Springer Science; 2006:159-182

66. Arias B: Diversidad de usos, prácticas de recolección y diferencia según género y edad en el uso de plantas medicinales en Córdoba, Argentina. Boletín Latinoamericana y del Caribe de Plantas Aromáticas 2009, 8:389-401.

67. Godoy R: The effects of rural education on the use of the tropical rainforest by the Sumu Indians of Nicaragua: possible pathways, qualitative findings, and policy options. Human Organization 1994 53:233-244

68. Reyes-García V, Valdez V, Huanca T, Leonard W, McDade T: Economic development and local ecological knowledge: A deadlock? Quantitative research from native Amazonian society. Hum Ecol 2007, 35:371-377.

69. Lynam T, Brown K: Mental models in human-environment interactions: theory, policy implications, and methodological explorations. Ecology and Society 2011, 17(3):24

70. Phillips O, Gentry A: The useful plants of Tambopata, Perú Il: additional hypothesis testing in quantitative ethnobotany. Econ Bot 1993, 47:33-43.

71. Albuquerque UP, Lucena RFP, Monteiro JM, Florentino ATN, Almeida CFCBR: Evaluating two quantitative ethnobotanical techniques. Ethnobot Res Appl 2006, 4:51-60.

doi:10.1186/1746-4269-10-14

Cite this article as: Beltrán-Rodríguez et al:: Factors affecting

ethnobotanical knowledge in a mestizo community of the Sierra de Huautla Biosphere Reserve, Mexico. Journal of Ethnobiology and Ethnomedicine 2014 10:14

\section{Submit your next manuscript to BioMed Central and take full advantage of:}

- Convenient online submission

- Thorough peer review

- No space constraints or color figure charges

- Immediate publication on acceptance

- Inclusion in PubMed, CAS, Scopus and Google Scholar

- Research which is freely available for redistribution

Submit your manuscript at www.biomedcentral.com/submit
C) Biomed Central 